\title{
ELECTRON CORRELATION PHENOMENA IN SELECTED TRANSITION METAL OXIDES
}

\author{
J.M. HONIG \\ Department of Chemistry, Purdue University, West Lafayette, Indiana 47907-1393, USA
}

The theory of electron correlation phenomena is reviewed and used to interpret the transport, thermal, or magnetic properties of $\mathrm{V}_{2} \mathrm{O}_{3}, \mathrm{LaNiO}_{3}$ and $\mathrm{Fe}_{3} \mathrm{O}_{4}$ systems, each of which to a greater or lesser degree displays correlation effects. A rationale is provided for interpreting the various types of phase transitions encountered in the vanadium sesquioxide and magnetite host systems and to show why these are altered by small compositional variations.

PACS numbers: 71.28.+d, 71.30.th, 75.10.Lp

\section{Introduction}

Certain classes of transition metal oxides represent systems in which electron correlation phenomena dominate or play an important role in determining their physical properties. We briefly review the experimental situation for the $\mathrm{V}_{2} \mathrm{O}_{3}$, $\mathrm{LaNiO}_{3}$, and $\mathrm{Fe}_{3} \mathrm{O}_{4}$ systems, where one frequently encounters spectacular manifestations of correlation phenomena, and then sketch out the basic theoretical framework by which these experimental results can be rationalized. Space limitations mandate a tight review pattern; for more extensive background information the reader is referred to earlier reviews on the subject matter $[1,2]$.

\section{The $\mathrm{V}_{2} \mathrm{O}_{3}$ alloy system}

The experimental situation in the $\left(\mathrm{V}_{2-x} \mathrm{M}_{x}\right)_{2} \mathrm{O}_{3}$ alloy system is perhaps best summarized by the data presented in Figs. $1 \mathrm{a}$ and 2 [3], which show the variation of the resistivity $\rho$ with temperature $T$ in a series of plots of $\log \rho$ vs. $1 / T$ for $\mathrm{M}=\mathrm{Cr}, \mathrm{Ti}$, and $\square$ (where $\square$ stands for cation vacancies generated when excess oxygen is introduced into the lattice). The measurements were carried out on single crystals grown by the skull melting technique [4], and subsequently annealed under appropriate oxygen atmospheres to control the oxygen/metal ratio. This work represents an extension of earlier, less comprehensive work by the Bell group [5] in this area; many other research groups have contributed additional insights, as documented in earlier reviews $[1,2]$. 

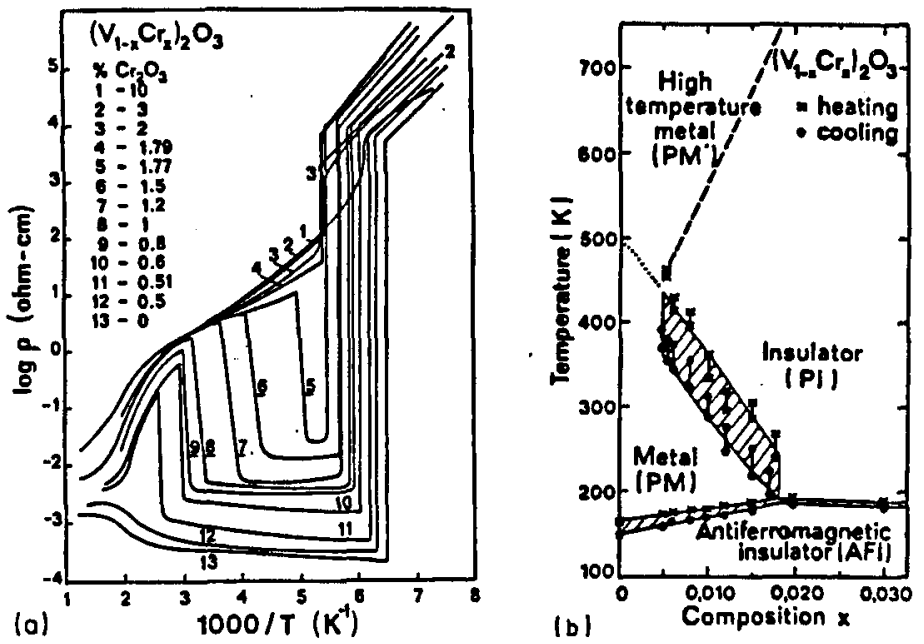

Fig. 1. (a) Measured resistivity $(\rho)$ curves for $\left(\mathrm{V}_{1-x} \mathrm{Cr}_{x}\right)_{2} \mathrm{O}_{3}$ plotted as $\log \rho$ vs. $1 / T$ for $\mathrm{V}_{2} \mathrm{O}_{3}$ with various $\mathrm{Cr}$ content. Note the many different types of phase transition obtained with small compositional variations. (b) Corresponding phase diagram for the $\left(\mathrm{V}_{1-x} \mathrm{Cr}_{x}\right)_{2} \mathrm{O}_{3}$ alloy system. Hatched areas represent hysteresis loops. Dashed and dotted lines represent approximate location of boundaries where high temperature metallic is separated by second order transitions from paramagnetic metallic and paramagnetic insulating phases. After Ref. [3].

The most striking features in Figs. 1 and 2 are the many metal-insulator transitions (MIT) that arise simply by varying the temperature or slightly altering sample compositions. The resulting experimental phase diagram is depicted in Fig. 1b. Corresponding drastic changes are seen in virtually all other physical properties: structure, optical characteristics, magnetic susceptibilities, thermal properties, and the like $[1,2]$. Up to three transitions occur with changes in temperature, depending on the nature and concentration of the element $\mathrm{M}$. This immediately raises the question as to how the $\mathrm{V}_{2} \mathrm{O}_{3}$ system can undergo such drastic alterations in response to very small energy inputs of the order of $k_{\mathrm{B}} T$ ( $k_{\mathrm{B}}$ represents Boltzmann's constant). Many different answers, listed in Refs. [1-3], have been proposed, on the basis of a very large body of disparate experimental observations. Note also that $\mathrm{V}_{2} \mathrm{O}_{3}$ with sufficient excess oxygen or titanium substitution remains a metal down to the cryogenic temperature range. Quite likely a combination of several schemes must be invoked for a complete accounting of all experimental results. Nevertheless, there is one telling experimental clue shown in Fig. 3, taken from experimental work by the Bell group [6]: heat capacity $\left(C_{p}\right)$ measurements were performed on $\mathrm{V}_{2} \mathrm{O}_{3}$ with sufficient excess oxygen or titanium to remain metallic down to lowest temperatures. Such data, plotted as $C_{p} / T$ vs. $T^{2}$, yield a straight line, with intercept $\gamma=90$ to $180 \mathrm{~mJ} /\left(\mathrm{K}^{2} \mathrm{~mole}\right)$ [6]. These data are consistent with the superposition of the Sommerfeld and Debye models for the heat capacity of solids in the low $T$ range. However, the electronic contributions 

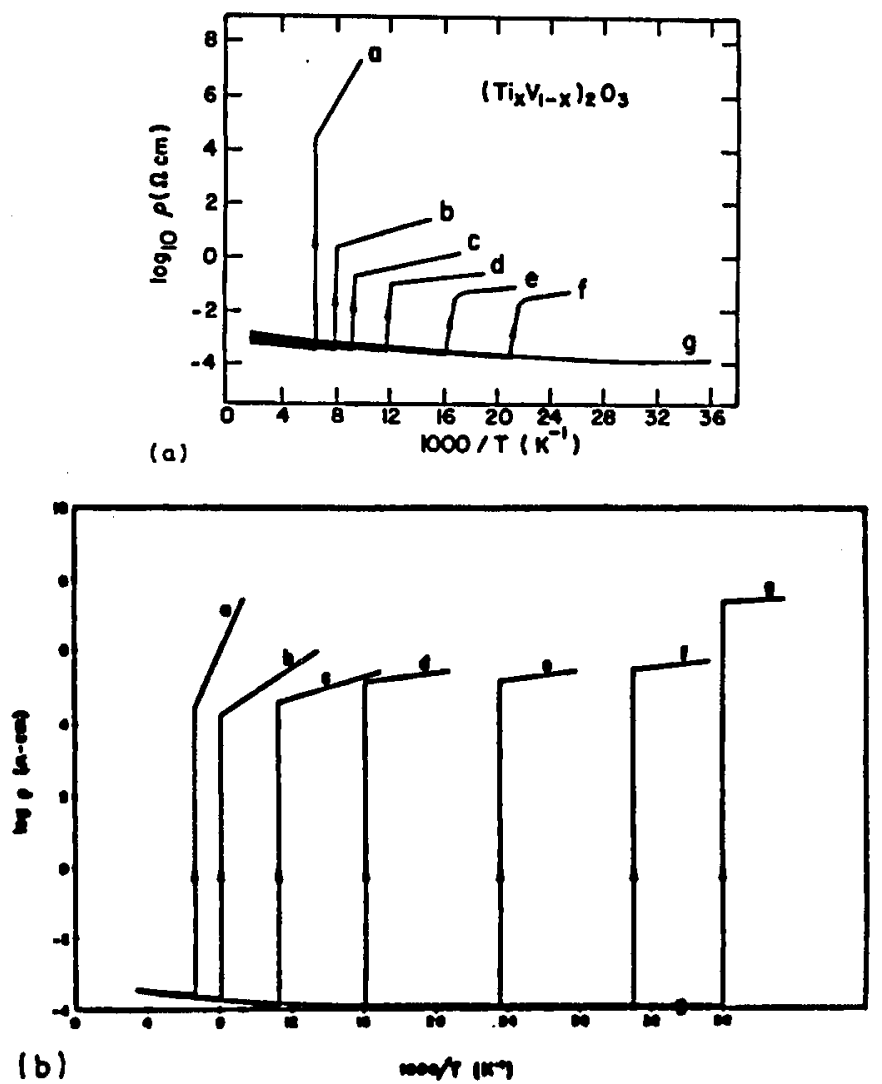

Fig. 2. (a) Measured resistivity curves for the $\left(\mathrm{V}_{1-x} \mathrm{Ti}_{x}\right)_{2} \mathrm{O}_{3}$ system, plotted as $\log \rho$ vs. $1 / T$. The $x$ values are $a, 0 ; b, 0.01 ; c, 0.02 ; d, 0.03 ; e, 0.04 ; f, 0.049 ; g, 0.055$. (b) Same plots for the $\mathrm{V}_{2(1-y)} \mathrm{O}_{3}$ system. The values are $a, 0 ; b, 0.0045 ; c, 0.0055$; $d, 0.0065 ; e, 0.0072 ; f, 0.0075 ; g, 0.0075+; h, 0.0095+$. Note the reduction in transition temperature with increasing $x$ or $y$. Beyond a critical composition the antiferromagnetic insulating phase is suppressed. After Ref. [3].

$\gamma$ to the specific heat is one order of magnitude larger than that of conventional metals such as $\mathrm{Cu}$. As discussed below, such high $\gamma$ values are symptomatic of strong electron correlation effects. The Purdue-Chicago group [3] verified the existence of a heat capacity peak in the 7-12 $\mathrm{K}$ range [6] which reflects the onset of antiferromagnetic ordering in the metallic phase; this feature was extensively studied in magnetic susceptibility measurements by Ueda et al. [7]. As also shown in Fig. 3, the resistivity $\rho_{e}$ of $\mathrm{V}_{2} \mathrm{O}_{3}$ under sufficient pressure to remain metallic varies with temperature $T<50 \mathrm{~K}$ as $\rho_{\mathrm{e}} \sim T^{2}$, which is consistent with the dominance of electron-electron scattering. These data show the close connection between electron correlation and magnetic ordering effects in the metallic phase. The corresponding situation for insulators is documented by the PM-AFI transi- 


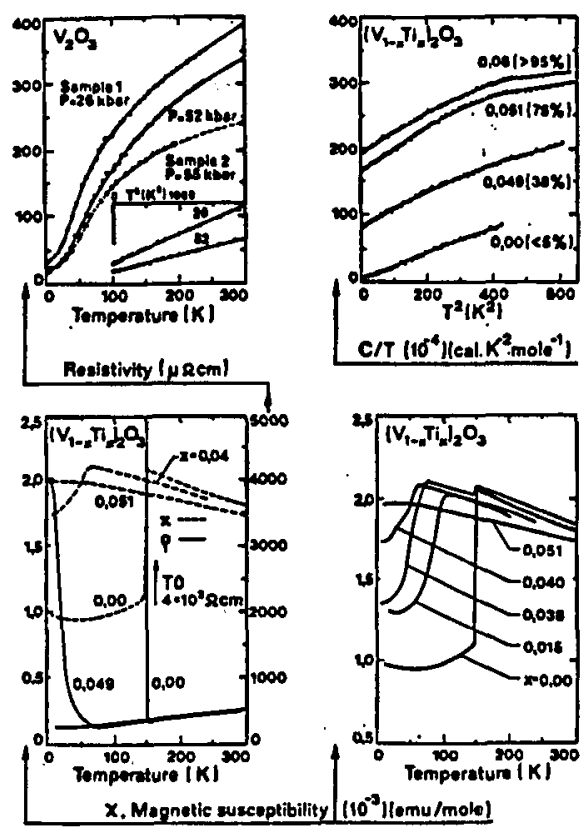

Fig. 3. Heat capacity, resistivity, and magnetic susceptibility measurements for $\mathrm{V}_{2} \mathrm{O}_{3}$ under pressure and for $\left(\mathrm{V}_{1-x} \mathrm{Ti}_{x}\right)_{2} \mathrm{O}_{3}$, collected by McWhan et al. [6].

tions below $170 \mathrm{~K}$ shown in Fig. 1. Contrary to the earlier stance taken by ours [3] the above results, and in particular, the reentrant metallic behavior, as well as a large body of additional evidence, can most readily be rationalized by invoking electron correlation effects. However, data presented later clearly show that correlations alone cannot deal with all observations; lattice effects and electron-phonon interactions also play a very important role.

Phase transitions of the type shown in Fig. 1 were originally discussed in terms of the Mott or Hubbard-Mott model [5, 6]. Here one envisions changes in physical properties brought about by alterations in lattice parameters or transfer integrals. In actuality, most experiments are performed on samples of fixed composition by varying temperature. To deal with such measurements one would have to render the relevant parameters (such as the intraatomic Coulomb repulsion energy $U$ ) temperature-dependent. This unsatisfactory situation was remedied by Spalek and his school [8] by explicitly introducing temperature as a variable in the construction of a free energy functional which is then minimized. The general procedure is briefly sketched below at an elementary level. For a proper discussion and for details the reader is referred to the original literature [8].

Basically, charge carriers in solids are subdivided into itinerant and localized sets. The latter designation is somewhat misleading; given sufficient thermal energy, charge carriers can move to adjacent sites either by thermal activation or by tunnelling. If the energy level of target site is already occupied by another electron, additional energy is required to overcome the intraatomic Coulomb repulsion $U$. 
The energy of localized charge carriers occupying individual sites in a nondegenerate band may be taken as the zero reference level, $E_{l}=0$. The entropy $S_{l}$ involves terms of the type $\sum_{i} P_{i} \ln P_{i}$, where $P_{i}$ is the probability that a representative site is in state $i$; here, $i$ indexes unoccupied, singly occupied, or doubly occupied levels. In the most interesting case $n=1$, with exactly one electron per site at $T=0$, the entropy per carrier is given by $\bar{S}_{l}=k_{\mathrm{B}} \ln 2$, because at every site the electron can be in either the spin-up $(\alpha)$ or spin-down $(\beta)$ configuration. This presumes that no magnetic ordering prevails. The free energy of the localized electron assembly is given by $F_{l}=E_{l}-T S_{l}$, and the reduced Helmholtz free energy per particle for an assembly of $N$ fermions is then ( $W$ is the band width for noninteracting electrons)

$$
F_{l} / W N=-\left(k_{\mathrm{B}} T / W\right) \ln 2 .
$$

The itinerant carriers are treated as an almost localized Fermi liquid constituted of quasiparticles, a feature that is justified a posteriori below. The heat capacity is then given by the Sommerfeld relation $C / N=\gamma T$, where $\gamma$ is yet to be determined in terms of $\gamma_{0} \equiv 2 \pi^{2} k_{\mathrm{B}}^{2} \rho_{0}^{\sigma}(\mu) / 3$, the linear specific heat coefficient for noninteracting electrons. Here $k_{\mathrm{B}}$ is Boltzmann's constant and $\rho_{0}^{\sigma}(\mu)$ is the density of states (DOS) per site per spin for bare electrons with energy $\mu$ at the Fermi level. Integration yields the expression for the energy $E_{i} / N=E_{0 i} / N+\frac{1}{2} \gamma T^{2}$, where $E_{0 i}$ is the energy of the interacting itinerant electron assembly at $0 \mathrm{~K}$.

To determine $E_{0 i}$ Spalek and coworkers [8] postulated that $E_{k}$, the energy of the quasiparticles with wave vector $\boldsymbol{k}$, is related to the corresponding energy $\varepsilon_{\boldsymbol{k}}$ of noninteracting electrons by a scaling relation of the form $E_{k}=\Phi(\eta) \varepsilon_{k}$, where $\Phi$ is an as yet unknown function of the probability $\eta \equiv\left\langle n_{i \uparrow} n_{i \downarrow}\right\rangle$ that any given site is doubly occupied by electrons with paired spins; $n_{i \sigma}$ is the corresponding number operator. Then the energy of the itinerant set is given by

$$
E_{\mathrm{B}} / N=\int_{-\infty}^{\infty} E f(E) \rho(E) \mathrm{d} E
$$

where $f(E) \equiv f\left[\left(E-E_{\mathrm{F}}\right) / k_{\mathrm{B}} T\right]$ is the Fermi-Dirac distribution function for the quasiparticles with the Fermi energy $E_{\mathrm{F}}$. On introducing the scaling law one obtains $f(\Phi \varepsilon) \equiv f\left[\Phi(\varepsilon-\mu) / k_{\mathrm{B}} T\right]=f\left[(\varepsilon-\mu) / k_{\mathrm{B}} T^{*}\right] \equiv f^{*}(\varepsilon)$, in which $\mu \equiv E_{\mathrm{F}} / \Phi$ is the Fermi energy for bare particles and $T^{*}=T / \Phi$ is an effective temperature. By definition, the DOS is given by $N \rho(E)=\sum_{(k)} \delta\left[\Phi\left(\varepsilon-\varepsilon_{k}\right)\right]=$ $\sum_{(k)} \Phi^{-1} \delta\left(\varepsilon-\varepsilon_{k}\right)=\Phi^{-1} \rho^{0}(\varepsilon) N$, so that $\rho^{\sigma}(E)=\Phi^{-1} \rho_{0}^{\sigma}(\varepsilon)$. We also replace $E$ by $\Phi(\eta) \varepsilon$ in Eq. (2). This yields

$$
E_{\mathrm{B}} / N=\Phi \int_{-\infty}^{\infty} \varepsilon f^{*}(\varepsilon) \rho_{0}^{\sigma}(\varepsilon) \mathrm{d} \varepsilon=\Phi(\eta) \bar{\varepsilon}\left(T^{*}\right) .
$$

Thus, the band energy of the quasiparticles has been reexpressed in terms of the kinetic energy $\bar{\varepsilon}\left(T^{*}\right)$ of noninteracting electrons at the effective temperature $T^{*}$ through the ubiquitous function $\Phi(\eta)$ which, in (3), is seen to play the role of an effective band narrowing factor.

We adjoin to (3) the potential energy of the interacting electron assembly. In the spirit of the Hubbard model the quasiparticles are assumed to interact only if they are located in pairs on the same atomic site. Let $U$ be the corresponding 
intraatomic potential energy; then the potential interaction energy per pair is given by $E_{\mathrm{P}}=U \eta$. Accordingly, the total energy per particle is

$$
E_{0 i} / N=\Phi(\eta) \bar{\varepsilon}\left(T^{*}\right)+U \eta
$$

To find $\Phi(\eta)$ we expand this function in powers of $\eta: \Phi(\eta)=f_{0}+f_{1} \eta+$ $f_{2} \eta^{2}+\ldots ;$ for a half-filled band with random occupancy $\eta=\left(\frac{1}{2} n\right)^{2}=1 / 4$, so that the expansion may be truncated as shown. The coefficients $f_{i}$ are found by optimizing $E_{0 i}$ and requiring $E_{0 i}$ to coincide with certain limiting cases for which this quantity is well established; for details consult Ref. [8]. One obtains the following result [9], valid for a half-filled band $(n=1)$ :

$$
\Phi(\eta)=8 \eta(1-2 \eta)
$$

Lastly, because of the assumed form $C / N=\gamma T$ for the quasiparticles, with $\gamma=2 \pi^{2} k_{\mathrm{B}}^{2} \rho^{\sigma}\left(E_{\mathrm{F}}\right) / 3=2 \pi^{2} k_{\mathrm{B}}^{2} \rho_{0}^{\sigma}(\mu) / 3 \Phi(\eta)$, we obtain $\gamma=\gamma_{0} / \Phi(\eta)$. The total energy of the itinerant quasiparticle assembly is then given by $E_{i} / N=\int \gamma T \mathrm{~d} T$, so that

$$
E_{i} / N=\Phi(\eta) \bar{\varepsilon}(0)+U \eta+\frac{1}{2}\left[\gamma_{0} / \Phi(\eta)\right] T^{2}
$$

The entropy is found from

$$
S_{i} / N=\int(\gamma / T) \mathrm{d} T=\left[\gamma_{0} / \Phi(\eta)\right] T,
$$

and the corresponding free energy is $(\bar{\varepsilon}(0) \equiv \bar{\varepsilon})$ :

$$
\begin{aligned}
F_{i}(T) / N & =E_{0 i} / N-\frac{1}{2} \gamma T^{2}=\Phi(\eta) \bar{\varepsilon}+U \eta-\frac{1}{2} \gamma_{0} \Phi(\eta)\left(T^{*}\right)^{2} \\
& =\Phi(\eta) F_{i}\left(T^{*}\right)^{2} / N+U \eta,
\end{aligned}
$$

which provides the a posteriorijustification for introducing the quasiparticle model: the free energy $F_{i}$ of the interacting particle assembly at temperature $T$ is related to that of a noninteracting electron assembly $F_{i}$ at temperature $T^{*}$ as shown in (8): $F_{i}\left(T^{*}\right)$ is multiplied by the band narrowing factor, and the Hubbard potential energy $U \eta$ is added to obtain $F_{i}(T)$. Having invoked the Sommerfeld approximation, the use of Eqs. (6)-(8) is limited to the low temperature regime $T^{*} \rightarrow 0$. One should note that $F_{i}(T)$ depends parabolically on $(T / \Phi)^{2}$ whereas, according to Eq. (1), $F_{l}$ varies linearly with $T$.

At this point one can provide a simple rationalization of the reentrant metallic behavior pattern encountered in the $\mathrm{V}_{2} \mathrm{O}_{3}$ system above $170 \mathrm{~K}$. Figure 4 shows a plot of $F_{i} / W N$ vs. $k_{\mathrm{B}} T / W$ both for the itinerant (Eq. (8)) and for the localized (Eq. (1)) models; curves $a-d$ are parabolas with different intercepts $E_{0 i} / N=-\Phi|\bar{\varepsilon}|+U \eta=-W \Phi / 4+U \eta$ based on use of a rectangular density states [RDOS]; the scheme applies for a half-filled band of energies in the range $-W / 2 \leq \varepsilon \leq W / 2$, for which the average kinetic energy is $\bar{\varepsilon}=-W / 4$. Line $e$ corresponds to Eq. (1). When $E_{0 i} / W N$ is sufficiently negative, i.e. for small values of $U \eta / W$ relative to $-\Phi|\bar{\varepsilon}| / W$ the parabola always lies below the straight line. Thus, the free energy of the paramagnetic metallic phase (PM) is always lower than that of the paramagnetic insulating phase (PI); there is no transition. This reflects the situation encountered experimentally in curves $g$ and $h$ displayed in Figs. 2a and 


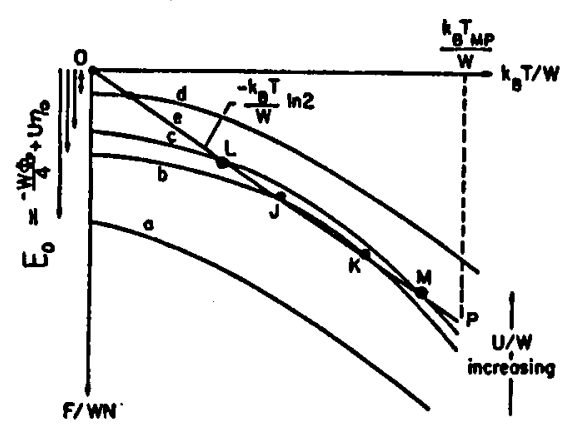

Fig. 4. Plot of $F_{i} / W N$ vs. $k_{\mathrm{B}} T / W$ for reduced free energies as specified in Eq. (1) for localized carriers and in Eq. (8) for itinerant quasiparticles. Here $W$ is the band width for noninteracting electrons and $U$ is the intraatomic electron repulsion energy. Note that the intercept for the parabolic curves becomes progressively 'more positive as $U / W$ increases.

$2 \mathrm{~b}$ respectively. As $U / W$ increases the intercept becomes less negative and the parabola first touches the straight line at one point and then intersects the latter at two points ( $J K$ or $L M$ in curves $b$ and $c$ in Fig. 4) that become increasingly separated with increasing $U / W$. Here the PM phase (parabolic curvature) has a lower free energy than the PI phase (linear variation) at the lowest and highest temperatures, but the reverse holds at intermediate temperatures. This situation reflects the regime of reentrant metallic behavior encountered above $180 \mathrm{~K}$ (curves 5-12 of Fig. 1) where the $\mathrm{PM} \rightarrow \mathrm{PI} \rightarrow \mathrm{PM}^{\prime}$ sequence of transitions is seen. As $U / W$ increases further the second intersection point moves to inaccessibly high temperature; only the $\mathrm{PI} \rightarrow \mathrm{PM}^{\prime}$ transition is encountered above $180 \mathrm{~K}$ (curves 1-4 of Fig. 1).

We return to Eq. (8); $F_{i} / N$ is seen to contain the extrathermodynamic variable $\eta$ which may now be determined by imposing the minimization constraint $\left(\partial F_{i} / \partial \eta\right)=0$. Straightforward manipulations yield the optimized expression for the probability of double occupancy of a given site to terms of order $T^{2}(n=1)$ :

$$
\eta_{\mathrm{opt}}=\eta_{0}-a T^{2}, a \equiv U \gamma_{0} / \Phi_{0}^{2} U_{c}^{2}, \eta_{0}=\frac{1}{4}\left(1-U / U_{\mathrm{c}}\right), U_{\mathrm{c}} \equiv 8|\bar{\varepsilon}| .
$$

The optimized band narrowing factor, determined from (5) becomes

$$
\left.\Phi\right|_{\mathrm{opt}}=\Phi_{0}-U a T^{2} /|\bar{\varepsilon}|, \quad \Phi_{0} \equiv 8 \eta_{0}\left(1-2 \eta_{0}\right)=1-\left(U / U_{\mathrm{c}}\right)^{2} .
$$

Then the reduced free energy reads

$$
F_{i}(T) / W N=-|\bar{\varepsilon}|\left(1-U / U_{c}\right)^{2} / W-\gamma_{0} T^{2} / 2 W\left[1-\left(U / U_{c}\right)^{2}\right],
$$

which is the optimized counterpart to Eq. (8).

Note that the Sommerfeld expansion applies only in the limit of low temperatures. We thus ignore the difference between $\mu(T)$ and $\mu(0) \equiv \mu_{0}$. We can then determine the PM-PI coexistence boundary by equating the corresponding partial molal free energies: $F_{l} / N=F_{i} / N$. On introducing Eqs. (1), (8), and (10) 
one obtains a quadratic equation in $k_{\mathrm{B}} T / W$ which may be solved for

$$
\frac{k_{\mathrm{B}} T_{ \pm}}{W}=\frac{3 \Phi_{0}}{2 \pi^{2} \rho^{0}(\mu) W}\left\{\ln 2 \pm\left[(\ln 2)^{2}-\frac{4 \pi^{2} \rho_{0}^{\sigma}\left(\mu_{0}\right)|\bar{\varepsilon}|}{3 \Phi_{0}}\left(1-\frac{U}{U_{c}}\right)^{2}\right]^{1 / 2}\right\} .
$$

Plots of $k_{\mathrm{B}} T / W$ vs. $U / U_{\mathrm{c}}$ lead to a retrograde curve of the type shown in Fig. $5 \mathrm{a}$. As shown elsewhere [10], the nature of these curves is not very sensitive to the choice of the DOS, $\rho_{0}^{\sigma}(\varepsilon)$. Figure $5 \mathrm{a}$ is based on the use of the rectangular density of states (RDOS) $\rho_{0}^{\sigma}=1 / W$ for $-W / 2 \leq \varepsilon \leq W / 2$, and 0 for $|\varepsilon|>W / 2$, where $\rho_{0}^{\sigma}$ is the DOS per site per spin.
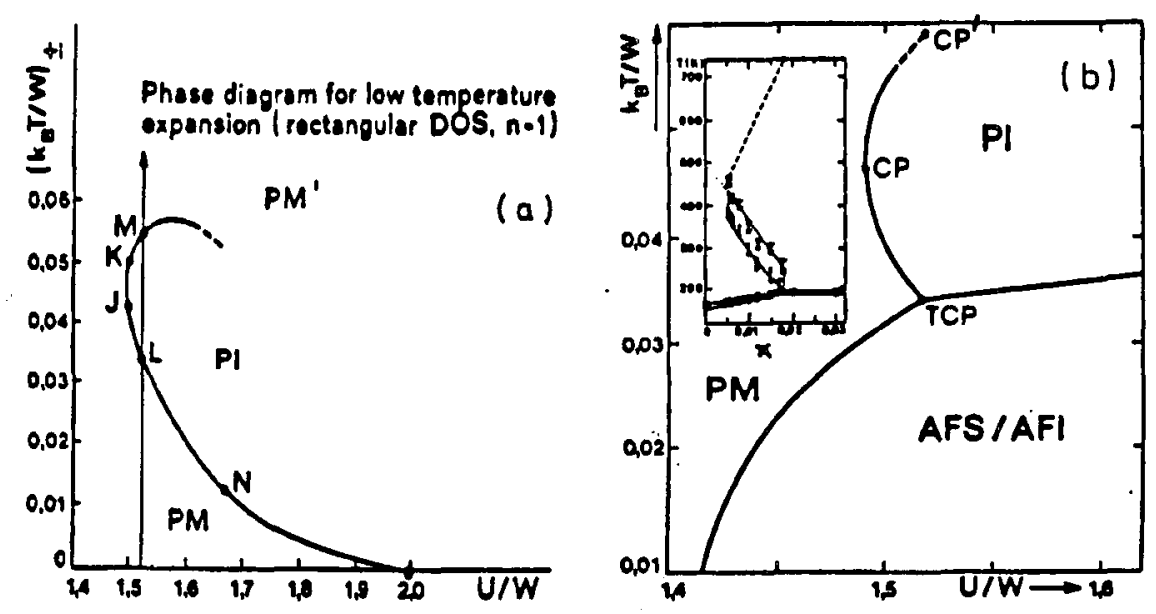

Fig. 5. Phase diagram for a correlated electron as manifested in the $\mathrm{V}_{2} \mathrm{O}_{3}$ host system. (a) Plot of reduced temperature vs. reduced intraatomic electron interaction energies, as specified by Eq. (12) with a RDOS; magnetic ordering effects are left out of account. The points $J K, L M$ correspond to the points so labelled in Fig. 4. (b) Phase diagram when magnetic ordering effects are taken into consideration; see text.

It should be noted that the curve displayed in Fig. $5 \mathrm{a}$ was truncated both at the top, where it began to turn over, and at the bottom, where it moved into the negative number domain. Both problems arise as a result of the approximations inherent in the theory. A more sophisticated two-fluid approach [11] avoids these difficulties; the curves are automatically truncated, as is documented in the original article.

One must now address the effects due to antiferromagnetic ordering at low temperatures. This is needed to account for the transitions displayed in Figs. 1-3 below $180 \mathrm{~K}$, because the low temperature phase is an antiferromagnetic insulator (AFI). The relevant theory is rather involved; for details the reader is referred to other sources $[2,13]$. Comparison of the Gutzwiller approach $[9,12]$ with Ref. [13] shows that in interacting electron systems charge carriers may be subdivided into localized and itinerant sets. In such a two-fluid model $\Phi$ represents as well the fraction of itinerant carriers. Only the portion $1-\Phi$ of the correlated 
metallic system $\left(U<U_{c}\right)$ is affected by the magnetic exchange effects. The latter are governed by the exchange constant $J \equiv\left(W^{2} / Z^{2} U\right)(1-\Phi)$ where $Z$ is the number of nearest neighbors to a given site. In the localized insulating phase $(\eta=\Phi=0)$ this reduces to $J=4 t^{2} / U$, where $t$ is the appropriate transfer integral. The general Hamiltonian operator now takes the form [13, 14]:

$$
\begin{aligned}
H= & \Phi \sum_{k \sigma} \varepsilon_{k} n_{k \sigma}+U \eta \\
& +J \sum_{j \in A} \sum_{j^{\prime}(j), j^{\prime} \in B}\left[S_{A j} \cdot S_{B j}-\frac{1}{4}\left(1-2 \eta_{i}\right)\left(1-2 \eta_{j}\right)\right],
\end{aligned}
$$

where $S_{j}, S_{j}$ are the spin operators for atoms of type $j, j^{\prime}$ on sublattices $A, B$ respectively, $j$ and $j^{\prime}$ being nearest neighbors; $\eta_{j} \equiv n_{j \uparrow} n_{j \downarrow}$.

The Hamiltonian (13) may be diagonalized by the Bogolyubov technique. The division of the lattice into two parts with exclusively spin-up and spin-down orientations yields a split band configuration [14] that results in a ground state reduced energy of the form $[13,14]$ :

$$
E_{\mathrm{m}} / W N=\frac{1}{4}\left[\Phi^{2}+\left(J Z\left\langle S^{2}\right\rangle / W\right)^{2}\right]^{1 / 2}+(U / W) \eta-(J Z / 8 W)(1-2 \eta)^{2}
$$

for a RDOS format. As $\Phi \rightarrow 0, \eta \rightarrow 0$ one converts from a Slater band insulator to the localized Mott insulator. The stability limit on the phase diagram may be determined by the coexistence condition $F_{\mathrm{m}}=F_{i}$; at low temperatures where the magnetic phase transition is encountered one assumes that $F_{\mathrm{M}} \approx E_{\mathrm{M}}$, because spin wave contributions $\left(\sim T^{4}\right)$ tend to be small. The reduced transition temperature is then specified by

$$
k_{\mathrm{B}} T_{\mathrm{tm}} / W=\left(2 \Phi / \gamma_{0}\right)^{1 / 2}\left[\bar{\varepsilon}\left(1-U / U_{\mathrm{c}}\right)^{2}-E_{\mathrm{m}} / N\right]^{1 / 2} .
$$

The final phase diagram for the $\left(\mathrm{V}_{1-x} \mathrm{Cr}_{x}\right)_{2} \mathrm{O}_{3}$ system is shown in Fig. 5b. The diagram is based on use of the RDOS and on the parameters $\mu_{\mathrm{m}}=1 \mu_{\mathrm{B}}, k_{\mathrm{B}} \theta_{N} / W=$ $J Z / 4 W=0.023$ and 0.031 for $U / W=1.65$ and 2 respectively; $W=1 \mathrm{eV}$. The inset shows the experimental phase diagram, the hatched areas delineate hysteresis effects. Arguments detailed elsewhere $[11,13]$ show that $U / W$ should scale as $x$ in $\left(\mathrm{V}_{1-x} \mathrm{Cr}_{x}\right)_{2} \mathrm{O}_{3}$. One sees the resemblance between calculated and experimental curves. In the present example we find that a tricritical point (TCP) is encountered under conditions where the PM, AFS/AFI (antiferromagnetic slater/antiferromagnetic insulator), and PI (paramagnetic insulator) phases coexist. More specifically, as $k_{\mathrm{B}} T / W$ increases one finds: (i) for $0<U / W<1.491$, only the AFS $\rightarrow$ PM transformation; (ii) for $1.491<U / W<1.517$, the AFS/AFI $\rightarrow \mathrm{PM} \rightarrow \mathrm{PI} \rightarrow \mathrm{PM}^{\prime}$ transition, which includes the reentrant metallic behavior; (iii) for $U / W>1.517$, the AFS/AFI $\rightarrow$ PI transformation, with a subsequent supercritical transformation to the more nearly $\mathrm{PM}^{\prime}$ configuration.

All of the various types of phase transitions encountered experimentally in the $\mathrm{V}_{2} \mathrm{O}_{3}$ system have thus been accounted for.

The above presentation should not obscure the fact that several important issues have not been addressed: the $\mathbf{P I} \rightarrow \mathrm{PM}^{\prime}$ phase transition is always gradual, contrary to the abrupt change predicted by the above theory; also, enormous hysteresis effects (of the order of $50 \mathrm{~K}$ ) are present at the PM $\rightarrow$ PI phase transformation. These phenomena clearly indicate that lattice effects cannot be ignored, 
which point has been repeatedly stressed by ours [3]. The role of the lattice has not yet been incorporated into the Spatek theory. Furthermore, within $10 \%$, the entropy of the PM $\rightarrow$ AFI transition can be accounted for by the standard relation $\Delta S_{\mathrm{V}}=R \ln (2 J+1)$, showing that magnetic ordering effects are largely responsible for this particular low-temperature transition [15]. Nevertheless, it is clear that electron correlation phenomena do play an important role in governing the phase transitions in the $\mathrm{V}_{2} \mathrm{O}_{3}$ systems.

\section{3. $\mathrm{LaNiO}_{3}$ as an example of a correlated metal}

$\mathrm{LaNiO}_{3}$ serves as a prototype example for displaying properties of a correlated metal. Although no single crystal measurements are available extensive sets of measurements on polycrystalline materials are generally in good quantitative agreement [16].

The variation with temperature in the resistivity $\rho_{l}$ of sintered bars is shown in Fig. 6a: below roughly $60 \mathrm{~K}, \rho_{l}=\rho_{0}+A T^{2}$, with $\rho_{0} \approx 0.5 \mathrm{~m} \Omega \cdot \mathrm{cm}$, $A=0.034 \mu \Omega \cdot \mathrm{cm} / \mathrm{K}^{2}$. The $\rho_{0}$ value likely is higher by one order of magnitude than that which would be measured in a single crystal, but even the extrapolated value indicates that the material is a poor metal. The $\rho_{l} \sim T^{2}$ dependence is generally interpreted in terms of the Baber-Landau scattering of electrons that form a Fermi liquid. The large value of the coefficient $A$ confirms that the electron scattering mechanism predominates at low temperatures and that correlation effects in $\mathrm{LaNiO}_{3}$ cannot be neglected.

The magnetic susceptibility $(\chi)$ variation with $T$ obtained by the Purdue group is shown in Fig. $6 \mathrm{~b}$; the inset is a greatly expanded $\chi$ plot in the range above $100 \mathrm{~K}$. These data can be fit by an expression of the form $\chi(T)=\chi(0)-$ $a T^{2}+C / T$, with $\chi(0)=5.1 \times 10^{-4} \mathrm{emu} / \mathrm{mole}, a=2.9 \times 10^{-10} \mathrm{emu} / \mathrm{K}^{2}$ mole, $C=3.4 \times 10^{-3}$ emu $\mathrm{K} / \mathrm{mole}$. As described below, the first and second terms characterize a slightly $T$-dependent Pauli paramagnetism, while the last term represents the Curie law with a small Curie constant. Whether the latter arises from residual impurities or is intrinsic is not known. However, the $\chi-T$ variation for $T<20 \mathrm{~K}$ cannot be quantitatively accounted for by the above relation. Lastly, Fig. 6c exhibits heat capacity measurements [16] in the range $0<T<10 \mathrm{~K}$; these can be fit to the relation $C_{p}=\gamma T+\beta^{\prime} T^{3}+\delta T^{3} \ln T$, with $\gamma=13.8 \mathrm{~mJ} / \mathrm{mole} \mathrm{K}^{2}$, $\beta^{\prime}=5.8 \times 10^{-5} \mathrm{~mJ} / \mathrm{K}^{3}$ mole, $\delta=0.12 \mathrm{~mJ} / \mathrm{K}^{3}$ mole. The first term, recognizable as the Sommerfeld contribution, is substantial, but it is smaller by a factor of three than that of $\mathrm{V}_{2} \mathrm{O}_{3}$ under pressure. The third term accounts for spin fluctuations [17] that produce a contribution of the type $\delta T^{3} \ln \left(T / T_{\mathrm{Sf}}\right)$. The $-\delta T^{3} \ln T_{\mathrm{Sf}}$ term has been combined with the Debye lattice contribution $\beta T^{3}$ to yield $\beta^{\prime} T^{3}$ in the second term. This example shows, incidentally, that $\beta^{\prime}$ can be quite small or even negative if the characteristic spin fluctuation temperature is substantial.

The theoretical analysis of the magnetic susceptibility requires a generalization of the central ansatz $E_{k}=\Phi \varepsilon_{k}$; namely, in an applied magnetic field $H$ the quasiparticle energy for state $k$ is given by $E_{k_{\sigma}}=\Phi_{\sigma}(\eta) \varepsilon_{k}-\sigma g \mu_{\mathrm{B}} H / 2$, where $\sigma= \pm 1$ is the spin variable, $g$ is the Lande factor, and $\mu_{\mathrm{B}}$ is the Bohr magneton. 

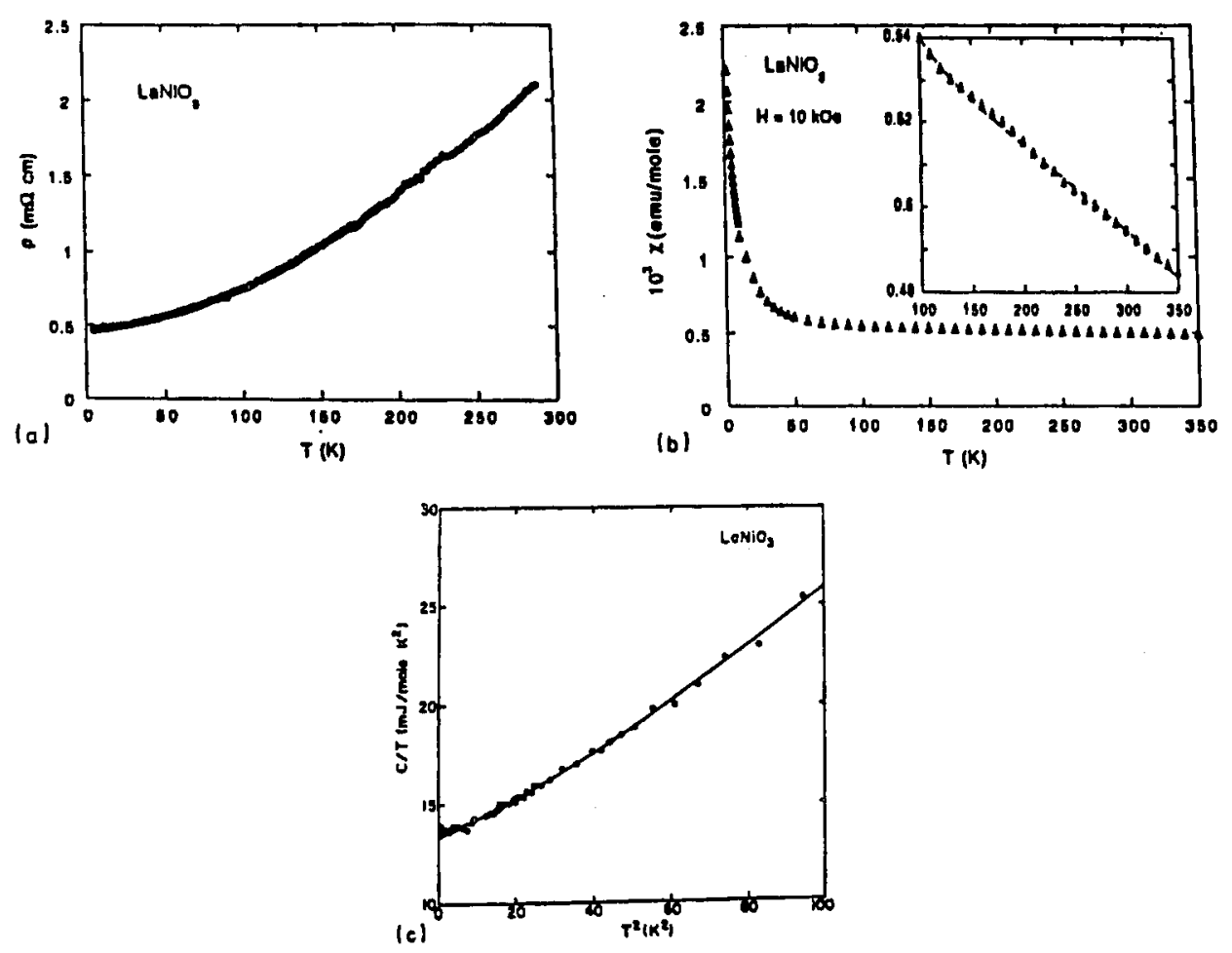

Fig. 6. (a) Temperature dependence of the resistivity of $\mathrm{LaNiO}_{3}$ ceramics; below $\approx 50 \mathrm{~K}$ $\rho \sim T^{2}$. (b) Temperature dependence of the molar susceptibility of $\mathrm{LaNiO}_{3}$ in an applied field of $10 \mathrm{kG}$. Inset shows the susceptibility variation at higher temperature on a greatly expanded $\chi$ scale; the dashed curve represents the theoretical fit. (c) Low-temperature specific heat of $\mathrm{LaNiO}_{3}$. The curve represents a fit to the relation $C / T=\gamma+\beta^{\prime} T^{2}+$ $\delta T^{2} \ln T$. Data reported by the Purdue group; after Ref. [16].

Using the above relation the free energy of the quasiparticle assembly is given by

$$
F / N=\sum_{\boldsymbol{k}_{\sigma}} E_{\boldsymbol{k}_{\sigma}}+U \eta+k_{\mathrm{B}} T \sum_{\boldsymbol{k}_{\sigma}}\left\{f_{\boldsymbol{k}_{\sigma}} \ln f_{\boldsymbol{k}_{\sigma}}+\left(1-f_{\boldsymbol{k}_{\sigma}}\right) \ln \left(1-f_{\boldsymbol{k}_{\sigma}}\right)\right\},
$$

where $f_{k_{\sigma}}$ is the Fermi-Dirac function written out below. The specification of the band narrowing factor in the presence of a magnetic field requires a lengthy analysis $[12,18]$ which is beyond the scope of the present review. For small magnetic fields one obtains

$$
\Phi(\eta, m)=8 \eta(1-2 \eta)\left\{1+m^{2}\left[1-1 / 4(1-2 \eta)^{2}\right]\right\},
$$

where $m=\left\langle n_{i \uparrow}-n_{i \downarrow}\right\rangle$ is the average magnetic polarization per site.

Equation (16) may be converted into a useful form by using arguments similar o those that led to (8); this involves use of the Sommerfeld expansion which applies only in the limit $T \rightarrow 0$. Additionally, we introduce the expression for the work per site in magnetizing the quasiparticle system. This quantity is given by

$$
W=\left(g \mu_{\mathrm{B}}\right)^{2} m^{2} / 8 \chi(T)=\left[\left(m g \mu_{\mathrm{B}}\right)^{2} / 8 \chi_{0}\left(T^{*}\right)\right] \Phi(\eta, m),
$$


where it is established below that $\chi(T)=\chi_{0}\left(T^{*}\right) / \Phi(\eta)$. On putting these results together one finds that [19]:

$$
\frac{F}{N}=\Phi(\eta, m) \bar{\varepsilon}+U \eta-\left[\gamma_{0} / 2 \Phi(\eta, m)\right] T^{2}+\left[\left(m g \mu_{\mathrm{B}}\right)^{2} / 8 \chi_{0}\left(T^{*}\right)\right] \Phi(\eta, m) .
$$

Equation (19) which is a generalization of Eq. (8) looks deceptively simple. One must actually specify several quantities for use in this relation:

(i) The DOS required in $\bar{\varepsilon}$ is given by $\rho^{\sigma}(E)=\rho_{0}^{\sigma}(\varepsilon) / \Phi(\eta, m)$, as before.

(ii) The Fermi-Dirac distributions function becomes

$$
\begin{aligned}
& f_{\sigma}(E)=\left\{1+\exp \left[E-\frac{1}{2} \sigma g \mu_{\mathrm{B}} H-\mu_{H}\right] / k_{\mathrm{B}} T\right\}^{-1} \\
&=\left.\left\{1+\exp \left[\varepsilon-\frac{1}{2} \sigma g \mu_{\mathrm{B}} H^{*}-\mu_{H}^{*}\right) / k_{\mathrm{B}} T^{*}\right]\right\}^{-1} \\
& \equiv f_{\sigma}\left[\left(\varepsilon-\frac{1}{2} g \mu_{\mathrm{B}} H^{*}-\mu_{H}^{*}\right) / k_{\mathrm{B}} T^{*}\right] \equiv f_{\sigma}(x),
\end{aligned}
$$

wherein $H^{*}=H / \Phi, T^{*} \equiv T / \Phi, \mu_{H}^{*}=\mu_{H} / \Phi, \varepsilon \equiv E / \Phi$. For small applied magnetic fields one may write

$$
f_{\sigma}(E) \equiv f\left[\frac{\varepsilon-\mu^{*}}{k_{\mathrm{B}} T^{*}}\right]-\left.\frac{1}{2} \frac{\sigma g \mu_{\mathrm{B}} H^{*}}{k_{\mathrm{B}} T^{*}} \frac{\partial f_{\sigma}}{\partial x}\right|_{x=\left(\varepsilon-\mu^{*}\right) / k_{\mathrm{B}} T^{*}} .
$$

(iii) The magnetization per particle in a small applied field is then given by

$$
\begin{aligned}
m & =\int \rho^{\sigma}(E)\left[f_{\uparrow}(E)-f_{\downarrow}(E)\right] \mathrm{d} E=\frac{g \mu_{\mathrm{B}} H}{\Phi} \int \mathrm{d} \varepsilon \rho_{0}^{\sigma}(\varepsilon) f^{\prime}\left(\frac{\varepsilon-\mu^{*}}{k_{\mathrm{B}} T^{*}}\right) \\
& =m_{0}\left(T^{*}, H^{*}\right),
\end{aligned}
$$

and the corresponding magnetic susceptibility reads

$$
\chi(T)=\frac{1}{2} g \mu_{\mathrm{B}} m=\chi_{0}\left(T^{*}\right) / \Phi(\eta, m),
$$

which indicates, incidentally that the magnetic susceptibility of an interacting particle assembly at temperature $T$ is related to that of the free electron assembly at temperature $T^{*}$ by the ubiquitous factor $\Phi(\eta, m)$. The above verifies a claim made in conjunction with Eq. (18).

(iv) The Fermi level is found via the constraint

$$
n_{\sigma} \equiv\left\langle n_{i \sigma}\right\rangle=\int \mathrm{d} E \rho^{\sigma}(E) f_{\sigma}(E)=\int \mathrm{d} \varepsilon \rho_{0}^{\sigma}(\varepsilon)\left[f_{\uparrow}(x)+f_{\downarrow}(x)\right] \text {. }
$$

(v) The required band narrowing function $\Phi(\eta, m)$, is specified by Eq. (17).

Equation (19) must now be optimized by imposing the requirement that $\partial F / \partial \eta=0$. This procedure yields an expression for the optimal double occupancy, $\eta_{\text {opt }}$, under the particular boundary conditions that have been imposed. The resulting expression is then substituted in (17) to obtain $\Phi_{\mathrm{opt}}=\Phi\left(\eta_{\mathrm{opt}}\right)$. To simplify matters Spałek and coworkers worked in the low temperature approximation: they introduced the general purpose integral $Q=2 \int g(\varepsilon) f(\varepsilon) \mathrm{d} \varepsilon$, where the function $g$ is dictated by the quantity of physical interest, and the integral is then expanded in ascending powers of $T$, using conventional techniques (see e.g. [20]). In the original work this derivation was carried through up to powers of $T^{4}$, but 
in the interest of simplicity only the terms in $T^{2}$ will be retained here, which is all that is required for the subsequent development.

We first consider the case of zero applied magnetic field $(m=0)$ for a nondegenerate half-filled band $(n=1)$. The condition $\partial F / \partial \eta=0$ is applied to Eq. (19), together with the expression $\partial \Phi / \partial \eta=8-32 \eta$ obtained from Eq. (17) with $m=0$. This procedure leads to the following set of results:

$$
\begin{aligned}
& \eta_{\mathrm{opt}}=\eta_{0}-a_{1} T^{2}, \\
& \Phi_{\mathrm{opt}}=\Phi_{0}-8 a_{1} I T^{2},
\end{aligned}
$$

with

$$
a_{1} \equiv \frac{2 \pi^{2}}{3} \frac{I \rho}{U_{\mathrm{c}} \Phi_{0}^{2}} k_{\mathrm{B}}^{2}
$$

where $\rho \equiv \rho_{0}^{\sigma}(\mu)$ is the DOS per site per spin at the Fermi level, and, as before,

$$
\Phi_{0} \equiv 1-I^{2}, I \equiv U / U_{\mathrm{c}}, U_{\mathrm{c}} \equiv 8|\bar{\varepsilon}|, \eta_{0}=\frac{1}{4}(1-I), \Phi_{0}=8 \eta_{0}\left(1-2 \eta_{0}\right) .
$$

Substitution of (25) with $m=0$ into (19) yields the earlier results

$$
F / N=(1-I)^{2} \bar{\varepsilon}-\gamma_{0} T^{2} / 2\left(1-I^{2}\right) \text {. }
$$

The heat capacity is then given by $C_{\mathrm{v}} / N=-T \partial^{2}(F / N) / \partial T^{2}$ :

$$
C_{\mathrm{v}} / N=\gamma_{0} T / 2\left(1-I^{2}\right)=\gamma_{0} T / \Phi_{0}, \quad \gamma_{0} \equiv 2 \pi^{2} k_{\mathrm{B}}^{2} \rho_{0}^{\sigma}(\mu) / 3 .
$$

The effective mass enhancement is found from the band narrowing factor:

$$
\frac{m^{*}}{m_{\mathrm{b}}}=\frac{1}{\Phi} \approx \frac{1}{1-I^{2}}\left[1+\frac{\gamma_{0} I^{2}}{|\bar{\varepsilon}|\left(1-I^{2}\right)^{3}}\right] \text {. }
$$

We note that both the contribution of charge carriers to the heat capacity as well as their effective masses become very large as $U \rightarrow U_{c}$, i.e., close to the metal-insulator transition. It is this feature that accounts for the sizeable $\gamma$ values reported in the $\mathrm{V}_{2} \mathrm{O}_{3}$ alloy system.

We next consider the magnetic susceptibility. We must now minimize the full functional, Eq. (19), with respect to $\eta$, in the limit of small $m$. The procedure is similar to that discussed in conjunction with Eqs. (25) but is more complex and can only be sketched out here. As a first step, one uses Eqs. (22) and (24), in which $\mu_{H}^{*} \equiv \mu_{H}\left(T^{*}, H^{*}\right)=\mu\left(T^{*}\right)+\Delta \mu\left(T^{*}, H^{*}\right)$ is expanded in powers of $g \mu_{\mathrm{B}} H^{*}$; use of Eq. (24) permits $\Delta \mu$ to be identified. One then determines the DOS by a Taylor expansion and one finds $\chi$ via Eq. (23) to obtain

with

$$
\chi\left(T^{*}\right)=\frac{1}{2}\left(g \mu_{\mathrm{B}}\right)^{2} \rho \alpha\left(T^{*}\right),
$$

$$
\alpha\left(T^{*}\right) \equiv 1-\frac{\pi^{2}}{6}\left[\frac{k_{\mathrm{B}} T}{\Phi}\right]^{2}\left[\left(\frac{\rho^{\prime}}{\rho}\right)^{2}-\frac{\rho^{\prime \prime}}{\rho}\right],
$$

where $\rho \equiv \rho_{0}^{\sigma}$ and its derivatives are to be evaluated at the Fermi energy.

Equations (29) and (17) are then introduced into Eq. (19) and the resulting full functional is subjected to the constraint $\partial F / \partial \eta=0$. One then obtains for small $m$ the following expression for the optimized double occupancy

$$
\eta_{\mathrm{opt}}=\eta_{0}-a_{1} T^{2}-a_{2} m^{2}-\ldots
$$


and for the corresponding band narrowing factor

$$
\Phi_{\text {opt }}=\Phi_{0}-8 I\left(a_{1} T^{2}+a_{2} m^{2}\right)-\ldots
$$

where higher order terms involving $m^{2} T^{2}$ have been dropped. Here

$$
a_{2} \equiv \frac{I}{16}\left\{\frac{1}{\rho|\bar{\varepsilon}|}+\frac{4(1-I)}{I(1+I)^{2}}-8 I \frac{1+I / 2}{(1+I)^{2}}\right\} .
$$

One should note that $\eta_{\mathrm{opt}}$ and $\Phi_{\mathrm{opt}}$ depend on $m^{2}$ and that these quantities appear in every term of Eq. (19). For this reason the susceptibility must be determined by use of Eq. (19) rather than being specified by the simple scaling procedure involving Eq. (29).

On now substituting (29) and (30) into (19) and differentiating the resultant twice with respect to the magnetic field one obtains (once more, $\rho \equiv \rho_{0}^{\sigma}(\mu)$ ):

$$
\begin{aligned}
\chi(T)) & =\left\{\Phi_{0}\left[1-\rho U(1+I / 2) /(1+I)^{2}\right]\right\}^{-1} \frac{1}{2}\left(g \mu_{\mathrm{B}}\right)^{2} \rho\left(1-B T^{2}\right) \\
= & \chi_{0}\left(1-B T^{2}\right) / \Phi_{0} S
\end{aligned}
$$

in which $B$ is a very complicated term involving $\rho$ and its derivatives, $U, U_{c}, I, \Phi_{0}$, and $a_{2}$. The above expression contains $S$, the renormalized Stoner factor, given by

$$
S \equiv 1-\rho U(1+I / 2) /(1+I)^{2} \text {. }
$$

Ordinarily $B T^{2} \ll 1$, so that in zero order one obtains a temperature-independent magnetic susceptibility $\chi_{0} / \Phi_{0} S$, which is obviously related to the Pauli paramagnetic susceptibility of uncorrelated metals $\chi_{0} \equiv \frac{1}{2}\left(g \mu_{\mathrm{B}}\right)^{2} \rho$. It is seen from Eq. (31a) that $\chi$ can grow without limit under two possible circumstances: (i) $I \rightarrow 1$ in the function $\Phi_{0}$; (ii) $\rho U(1+I / 2) /(1+I)^{2} \rightarrow 1$, which leads to the vanishing of the Stoner factor $S$. In the first case one encounters the Mott-Hubbard transition to a localized moment configuration; in the second case the system undergoes a magnetic phase transition. If the DOS at the Fermi energy is large, (ii) precedes (i), otherwise the reverse holds. It should be noted that for a RDOS with $\rho=1 / W, \rho U=2 I$, one finds $S=(1+I)^{-2} \geq \frac{1}{4}>0$, i.e. the RDOS is not large enough to induce a magnetic transition. In general, both the localization factor $\Phi_{0}^{-1}$ and the renormalized Stoner factor $S^{-1}$ contribute to an enhancement of the magnetic properties.

We are now in a position to apply the above theory to an interpretation of the data for $\mathrm{LaNiO}_{3}$. Namely, we set $\gamma=13.8 \mathrm{~mJ} /\left(\mathrm{mole} \mathrm{deg}^{2}\right)$ and $\chi=$ $0.61 \times 10^{-3} \mathrm{emu} / \mathrm{mole}$ to determine the ratio (which is the inverse of the so-called Wilson ratio)

$$
\frac{\gamma}{\chi}=\frac{\pi^{2} k_{\mathrm{B}}^{2}}{3 \mu_{\mathrm{B}}^{2}} S
$$

as taken from Eqs. (27) and (31a). This leads to a value $S=0.31$; one sees that the system is still rather far removed from a magnetic instability. For a RDOS, for which $S=(1+I)^{-2}$, one obtains the ratio $I=U / U_{\mathrm{c}}=0.79$, again rather far removed from an instability edge. The effective mass as specified by Eq. (28) leads to a value $m^{*} / m_{\mathrm{b}}=2.6$. From Eq. (27) one then obtains the RDOS value of $\rho \approx$ $1.2(\mathrm{eV} \text { site spin })^{-1}$, which yields $W=1 / \rho=0.87 \mathrm{eV}$ and $U=2 I W=1.67 \mathrm{eV}$. The above results indicate the $\mathrm{LaNiO}_{3}$ is a relatively narrow-band metal with a significant but not exceptionally large intraatomic Coulomb interaction. 


\section{The magnetite, titanomagnetite, and zinc ferrite system}

The magnetite system $\mathrm{Fe}_{3} \mathrm{O}_{4}$ has been the object of considerable study because of a phase transition that occurs near $120 \mathrm{~K}$, the so-called Verwey transition. The characterization of this phase transformation is the subject of the present section. Most of the early work is superseded by the recent recognition [21] that small changes in the oxygen to metal ratio, represented by $\delta$ in the formula $\mathrm{Fe}_{3(1-\delta)} \mathrm{O}_{4}$ $(-0.0005 \leq \delta \leq 0.012)$, completely alter the characteristics of the material. Figures 7 and 8 display typical changes in heat capacity and electrical resistivity $\rho_{\mathrm{e}}$

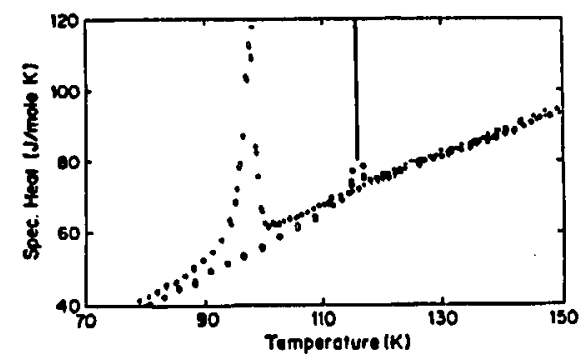

Fig. 7. Comparison of heat capacities for two samples exhibiting Verwey transitions of different order: $\delta=0.00021$, first-order transition, and $\delta=0.0049$, second- or higher-order transition. After Ref. [21].

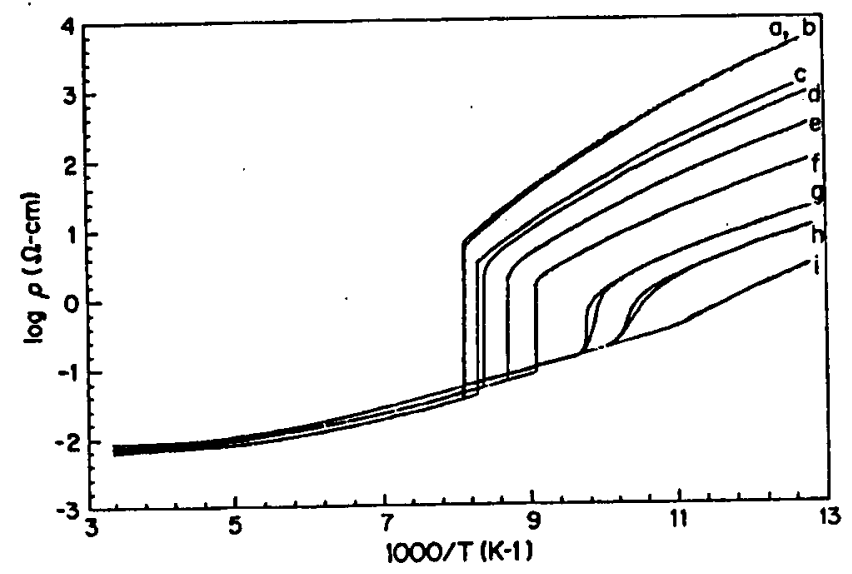

Fig. 8. Variation of electrical resistivity $(\rho)$ with temperature for $\mathrm{Fe}_{3(1-\delta)} \mathrm{O}_{4}$ single crystal specimens: $\delta=-0.00053(a),-0.00017(b), 0.00021(c), 0.00069(d), 0.0017(e)$, $0.0035(f), 0.0050(g), 0.0068(h), 0.009(i)$. After Ref. [21]. 

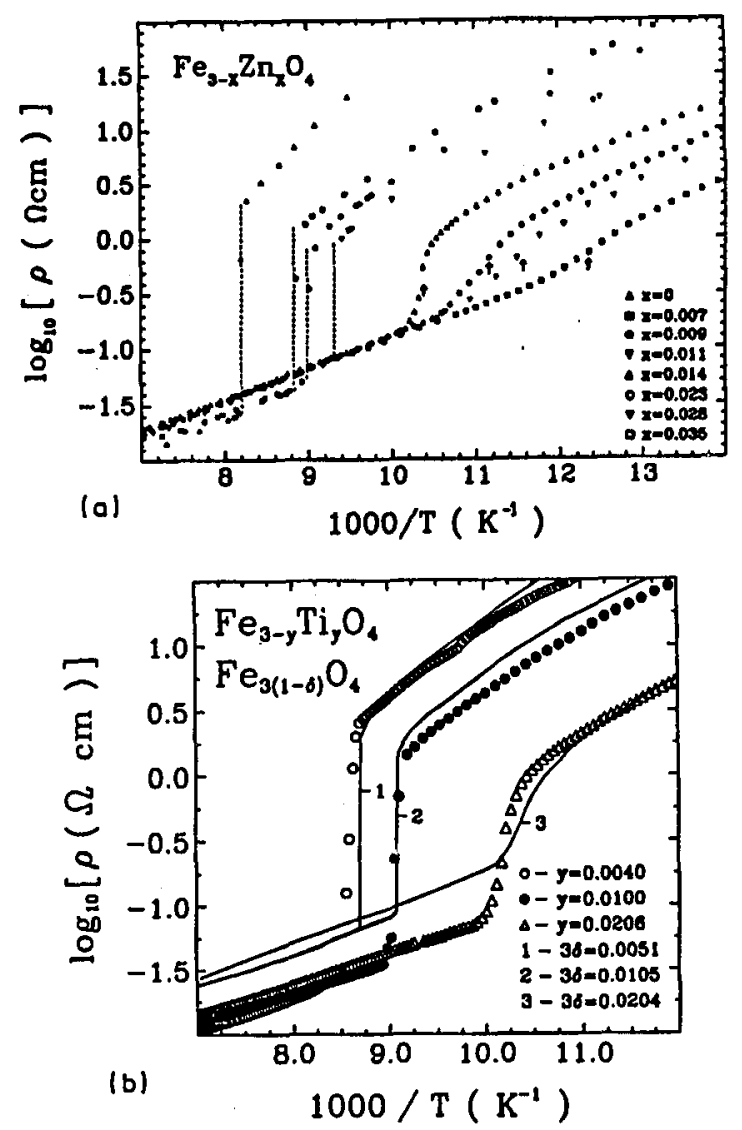

Fig. 9. (a) Composite plot of the variation of resistivity with temperature near the Verwey transition for zinc ferrites $\mathrm{Fe}_{3-x} \mathrm{Zn}_{x} \mathrm{O}_{4}$ with $0 \leq x \leq 0.035$. (b) Detailed comparison of electrical property of dilute titanomagnetites and nonstoichiometric magnetite near the Verwey transition; note the correspondence $y=3 \delta$. After Ref. [21].

encountered in specimens of different compositions $\delta$. In Fig. 9a and $9 \mathrm{~b}$ are shown examples of resistivity changes near the Verwey transition temperature for zinc ferrites samples of composition $\mathrm{Fe}_{3-x} \mathrm{Zn}_{x} \mathrm{O}_{4}$, and for the titanomagnetite series $\mathrm{Fe}_{3-y} \mathrm{Ti}_{y} \mathrm{O}_{4}$ [21]. With increasing $\delta, x$, or $y$ there are striking alterations in the nature of the Verwey transformation: it changes from first order to higher order and ultimately disappears. Correspondingly; these variations are authenticated by discontinuities in $\rho_{\mathrm{e}}$ and by a latent heat of transition in the composition range $-0.0005 \leq \delta<0.0039 \equiv \delta_{\mathrm{c}}$. An abrupt change in the derivative $\rho_{\mathrm{e}}^{\prime}(T)$ and no latent heat is encountered in the range $\delta_{\mathrm{c}}<\delta<3 \delta_{\mathrm{c}}$. The electrical properties of nonstoichiometric magnetite, zinc ferrites, and titanomagnetites match very closely, with the correspondence $3 \delta=x=y$. Furthermore, there are no resistivity anomalies for $\delta=x / 3=y / 3>3 \delta_{\mathrm{c}}$. This collection of regimes is summarized in a plot of the 


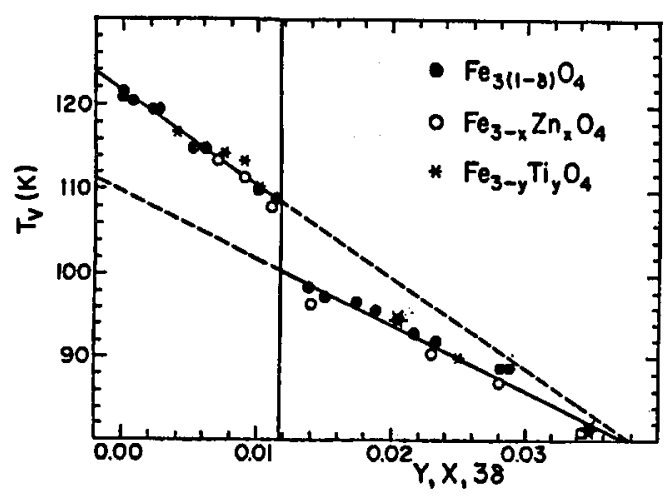

Fig. 10. Variation of Verwey transition temperature against sample composition for dilute titanomagnetite, zinc ferrite, or nonstoichiometric magnetite. The regions of firstand higher-order transitions are clearly delineated. Note the correspondence $x=y=3 \delta$.

Verwey transition temperature $T_{\mathrm{V}}$ vs. $3 \delta=x=y$ in Fig. 10, where the first and higher order transformation regimes are clearly evident. It is remarkable that very small changes in sample composition can bring about such drastic alterations in the nature of the thermodynamic properties of the material.

The challenge here is to find a single formalism that can handle the first and higher order transition in a unified scheme. At a very elementary level this may be done [22] through the recognition that magnetite and its dilute $\mathrm{Zn}$ and $\mathrm{Ti}$ based derivatives form an inverse spinel of the type $\left(\mathrm{Fe}^{3+}\right)\left[\mathrm{Fe}^{2+}, \mathrm{Fe}^{3+}\right] \mathrm{O}_{4}$ in which (..) and [ . .] denote tetrahedrally and octahedrally coordinated interstices in the cubic oxygen framework. In first approximation, the electrical characteristics are governed by the electron interchange involving "filled" $\left[\mathrm{Fe}^{2+}\right]$ sites containing six $d$ electrons and "empty" $\left[\mathrm{Fe}^{3+}\right]$ sites with five $d$ electrons, both interstices being octahedrally coordinated. The $\mathrm{Fe}^{3+}$ configuration will be considered to form the vacuum state, while the sixth electron is free to move. The energetically most favorable situation is that in which every $\mathrm{Fe}^{2+}$ is surrounded by $\mathrm{Fe}^{3+}$ and vice versa, but this is also the configuration of lowest entropy. Furthermore, the $\mathrm{Fe}^{2+} / \mathrm{Fe}^{3+}$ balance in the octahedral sites is disturbed either by introduction of excess oxygen (producing more $\mathrm{Fe}^{3+}$ at the expense of $\mathrm{Fe}^{2+}$ to preserve electroneutrality), or by substitution of $\mathrm{Zn}^{2+}$ for $\mathrm{Fe}^{3+}$ in the tetrahedral interstices (producing the same effect), or by incorporation of $\mathrm{Ti}^{4+}$ in the octahedrally coordinated interstices (generating $\mathrm{Fe}^{2+}$ at the expense of $\mathrm{Fe}^{3+}$ to maintain electroneutrality).

In these circumstances it is expedient as a rock-bottom approximation to replace the magnetite system by a collection of site pairs (o-0), also called "bonds", which may be in the occupation states shown in Table. The lowest energy state is that in which one electron is strictly localized at one of the two o-sites through a small-polaron trapping mechanism involving a lattice deformation around the self-trapped charge carrier. This deformation incurs a lattice distortion of the type involved during the transition from the cubic spinel $\left(T>T_{\mathrm{V}}\right)$ to the monoclinic $\left(T<T_{\mathrm{V}}\right)$ configuration. Such a state is schematized in Fig. 11a. The corresponding 
TABLE

Configurations of site pairs and sites.

\begin{tabular}{c|c|c|c}
\hline \hline Configuration & Designations & Probability & Energy \\
\hline$\otimes \longrightarrow$ & $C A$ & $\beta_{0}$ & $\varepsilon_{C A}$ \\
$\bullet-0$ & $B A$ & $\beta_{1}$ & $\varepsilon_{B A}$ \\
$\circ \longrightarrow$ & $A B$ & $\beta_{1}$ & $\varepsilon_{A B}$ \\
$\bullet-$ & $B B$ & $\beta_{2}$ & $\varepsilon_{B B}$ \\
$\otimes$ & $C$ & $\gamma_{0}$ & $\varepsilon_{C}$ \\
$\circ$ & $A$ & $\gamma_{1}$ & $\varepsilon_{A}$ \\
$\bullet$ & $B$ & $\gamma_{2}$ & $\varepsilon_{B}$ \\
\hline
\end{tabular}

There are $Z L / 2$ bonds (site pairs) and $(1-Z) L$ sites to represent the collection of $L$ octahedrally coordinated interstices with $Z=4$ nearest neighbors in magnetite.

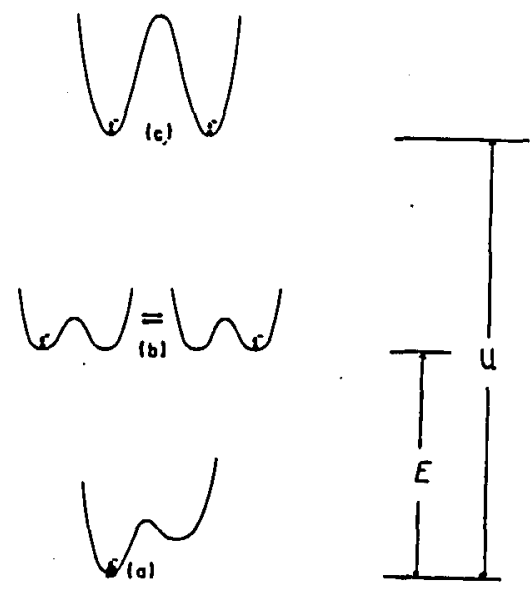

Fig. 11. Schematic diagrams of energy states of the bond figure assembly. (a) Ground state energy; the electron $\otimes$ is in a trapped state. (b) Resonant state which is a precursor to directed drifts in an applied electric field; • represents a mobile electron. (c) Two electrons in adjacent octahedrally coordinated interstices; the mobile charge carriers can jump to adjacent empty sites.

bond configuration is designated by $\otimes-0$, while the interchange to the $o-\otimes$ site is inadmissible. As shown in Table, the corresponding probability and energy is given by $\beta_{0}$ and $\varepsilon_{C A}$ respectively. A first excited state is generated by promoting charge carriers to the resonant configuration shown in Fig. 11b; here the carrier can resonate between two equivalent states depicted by $\bullet-\circ$ and $\circ-\bullet$ respectively, with probabilities $2 \beta_{1}$ and energies $\varepsilon_{B A}=\varepsilon_{A B}$. The excess carrier associated with $\mathrm{Fe}^{2+}$ ions forms the precursor state to directed drift under an imposed electric field. 
The state of highest energy is depicted in Fig. 11c as arising when two electrons occupy adjacent octahedral sites; such carriers cannot move toward each other but they can proceed to other adjacent empty sites. This state is represented by $\bullet-\bullet$ and occurs with probability $\beta_{2}$ and has an associated energy $\varepsilon_{B B}$.

A total of $Z L / 2$ bonds are required to represent the octahedrally coordinated site pairs in the $\mathrm{Fe}_{3} \mathrm{O}_{4}$ lattice; here $L$ is the total number of sites and $Z$ is the number of nearest neighbors. This collection of bond figure assemblies already contains a total of $Z L$ individual sites, whereas the actual number of such sites is $L$. Accordingly, one introduces an assembly of $(1-Z) L$ additional single sites so that the overall total in the representative assembly matches the actual number in the lattice. The individual site occupation states are shown schematically, together with their associated probabilities and energies, at the bottom of Table. The three possibilities involve a fraction $\gamma_{0}$ of $\otimes$ sites occupied by electrons trapped through lattice deformations, with a corresponding energy $\varepsilon_{C}$. A fraction $\gamma_{1}$ of individual sites (o) is unoccupied; this quantity is determined by the composition of the solid. A fraction $\gamma_{2}$ involves sites $(\bullet)$ containing mobile charge carriers associated with an energy $\varepsilon_{B}$; the density of these carriers in principle is accessible via the Seebeck coefficient measurements.

This extremely simple model will be shown to suffice to handle all the complexities of the magnetite systems; improved treatments may be devised but they involve a much greater number of adjustable parameters.

The probabilities listed in Table are not independent. One must take into account the normalization requirements

$$
\begin{aligned}
& \beta_{2}+2 \beta_{1}+\beta_{0}=1, \\
& \gamma_{2}+\gamma_{1}+\gamma_{0}=1,
\end{aligned}
$$

and the consistency conditions for mobile electrons

$$
\beta_{2}+\beta_{1}=\gamma_{2},
$$

and the density of empty sites

$$
\gamma_{1} \equiv c \text {, }
$$

where $c$ is the density of $\mathrm{Fe}^{3+}$ ions as determined from the compositional parameters $\delta, x$, or $y$. We now arbitrarily select $\gamma_{2}$ and $\beta_{2}$ as the independent variables, and set $\beta_{1}=\gamma_{2}-\beta_{2}, \beta_{0}=1-2 \gamma_{2}+\beta_{2}, \gamma_{0}=1-c-\gamma_{2}$.

The energy of the bond figure assembly representing $\mathrm{Fe}_{3} \mathrm{O}_{4}$ is then given by

$$
E_{\mathrm{b}}=\frac{Z}{2} L\left(\beta_{2} \varepsilon_{B P}+2 \beta_{1} \varepsilon_{A B}+\beta_{0} \varepsilon_{C A}\right)=\frac{Z}{2} L\left(\beta_{2} U+2 \beta_{1} E+\varepsilon_{C A}\right),
$$

where $U \equiv \varepsilon_{B B}-\varepsilon_{C A}=U\left(\beta_{2}\right)$ and $E=\varepsilon_{B A}-\varepsilon_{C A}=E\left(\beta_{1}\right)$. The energies of the site figure assembly is written as

$$
E_{\mathrm{s}}=(1-Z) L\left(\gamma_{2} \varepsilon_{B}+\gamma_{1} \varepsilon_{A}+\gamma_{0} \varepsilon_{C}\right)=(1-Z) L\left(\gamma_{2} \varepsilon_{2}+\gamma_{1} \varepsilon_{1}+\varepsilon_{0}\right),
$$

where $\varepsilon_{2} \equiv \varepsilon_{B}-\varepsilon_{C}, \varepsilon_{1}=\varepsilon_{A}-\varepsilon_{C}$. The entropy in Stirling's approximation is given by

$$
S_{\mathrm{b}}=-k_{\mathrm{B}} \frac{Z}{2} L\left(\beta_{2} \ln \beta_{2}+2 \beta_{1} \ln \beta_{1}+\beta_{0} \ln \beta_{0}\right)
$$


for the bond figure assembly, and by

$$
S_{s}=-k_{\mathrm{B}}(1-Z) L\left(\gamma_{2} \ln \gamma_{2}+\gamma_{1} \ln \gamma_{1}+\gamma_{0} \ln \gamma_{0}\right)
$$

for the site figure assembly.

The Helmholtz free energy, $F=E-T S$ for the representative assembly may be determined from Eqs. (34) and (35). Equilibrium constraints are imposed by demanding that $\partial F / \partial \beta_{2} \equiv \partial F_{\mathrm{b}} / \partial \beta_{2}=0$. One must recall that $\beta_{1}$ and $\beta_{0}$ are also functions of $\beta_{2}$. We find

$$
\begin{aligned}
0= & \frac{Z}{2} L\left(\beta_{2} U^{\prime}+U+2 E \frac{\partial \beta_{1}}{\partial \beta_{2}}+2 \beta_{1} E^{\prime} \frac{\partial \beta_{1}}{\partial \beta_{2}}\right)+k_{\mathrm{B}} T \frac{Z}{2} L\left[\ln \beta_{2}+2\left(\ln \beta_{1}\right) \frac{\partial \beta_{1}}{\partial \beta_{2}}\right. \\
& \left.+\left(\ln \beta_{0}\right) \frac{\partial \beta_{0}}{\partial \beta_{2}}+1+2 \frac{\partial \beta_{1}}{\partial \beta_{2}}+\frac{\partial \beta_{0}}{\partial \beta_{1}}\right],
\end{aligned}
$$

which may be arranged in the form

$$
\ln \left(\beta_{2} \beta_{0} / \beta_{1}^{2}\right)=-\left[(U-2 E)+\beta_{2} U^{\prime}-2 \beta_{1} E^{\prime}\right] / k_{\mathrm{B}} T \equiv-R / k_{\mathrm{B}} T,
$$

or

$$
\beta_{2} \beta_{0} / \beta_{1}^{2}=\mathrm{e}^{-R / k_{\mathrm{B}} T} \equiv C
$$

The subsequent analysis is much simplified by considering two special cases. The first of these arises when $C=0$ under the sufficient condition that $U \rightarrow \infty$ in Eq. (37a). Equation (37b) shows that we can achieve this requirement by setting $\beta_{2}=0$, so that $\beta_{1}=\gamma_{2}$ and $\beta_{0}=1-2 \gamma_{2}$. The free energy of the bond figure assembly is now given by $\left(\tilde{F}_{\mathrm{b}} \equiv F_{\mathrm{b}} / L\right)$ :

$$
\tilde{F}_{\mathrm{b}}=2 \beta_{1} E+\varepsilon_{C A}+k_{\mathrm{B}} T\left[2 \beta_{1} \ln \beta_{1}+\left(1-2 \beta_{1}\right) \ln \left(1-2 \beta_{1}\right)\right] .
$$

We next introduce the order parameter $\psi \equiv 2 \beta_{1}$ to obtain

$$
\widetilde{F}_{\mathrm{b}}=\psi E(\psi)+\varepsilon_{C A}+k_{\mathrm{B}} T[\psi \ln \psi+(1-\psi) \ln (1-\psi)-\psi \ln 2] .
$$

Optimization with respect to $\psi$ via $\partial \widetilde{F}_{\mathrm{b}} / \partial \psi=0$ yields the equilibrium condition

$$
\frac{E(\psi)+\psi E^{\prime}(\psi)}{k_{\mathrm{b}} T}=-\ln \left(\frac{\psi}{1-\psi}\right)+\ln 2
$$

For the special case where one adopts a linear variation $E(\psi)=\varepsilon_{0}-\frac{1}{2} \lambda \psi$ one obtains

$$
\left(\varepsilon_{0}-\lambda \psi\right) / k_{\mathrm{B}} T=-\ln [\psi /(1-\psi)]+\ln 2 .
$$

One should note that Eqs. (39) and (40) provide a rationalization of the relationship first postulated by Strässler and Kittel [23] to deal with first order phase transitions; $g_{1} / g_{0} \equiv 2$ in Ref. [23].

Equation (40b) may be solved numerically for $\psi=\psi\left(T ; \varepsilon_{0}, \lambda\right)$ once the microscopic quantities $\varepsilon_{0}$ and $\lambda$ have been specified. The program of data fitting is discussed elsewhere in detail [24] and will not be repeated here; basically, one imposes conditions that must be met by the function $\widetilde{F}_{\mathrm{b}}$ and its derivatives at the transition point. Numerical calculations of $\psi\left(T ; \varepsilon_{0}, \lambda\right)$ are displayed by curves $a-f$ in Fig. 12; one should note the discontinuity in $\psi$ at $T=T_{\mathrm{V}}$, symptomatic of first order transitions.

As the opposite extreme we consider the case $C=1$, which corresponds to complete randomization, under the sufficient condition $U=2 E, U^{\prime}=E^{\prime}=0$ 


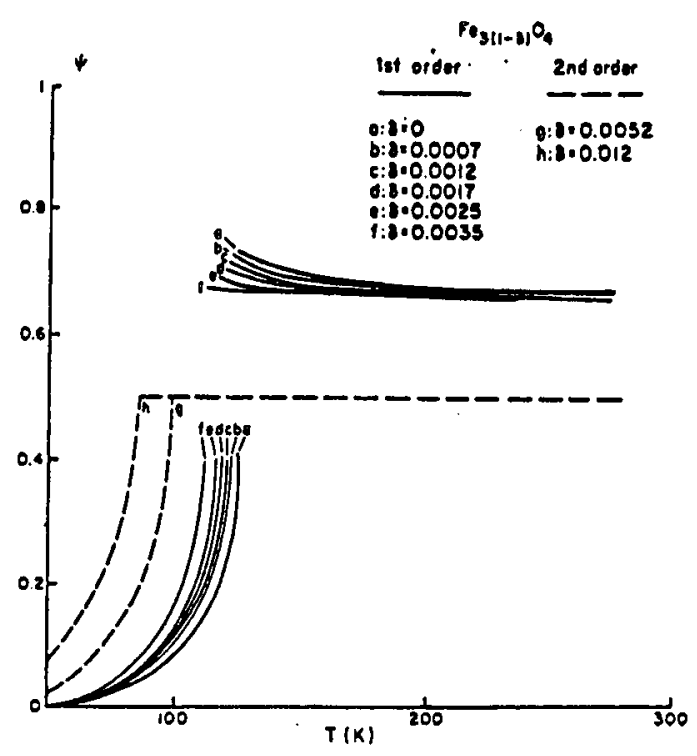

Fig. 12. Plots of order parameter $\psi$ versus temperature $T$ for the $\delta$ values indicated in the figure. Full curves: first-order regime. Dashed curves: second-order regime.

in Eq. (37a). One now obtains $\beta_{2}=\gamma_{2}^{2}, \beta_{1}=\gamma_{2}\left(1-\gamma_{2}\right), \beta_{0}=\left(1-\gamma_{2}\right)^{2}$ and a Helmholtz free energy of the form

$$
\widetilde{F}_{\mathrm{b}}=2 E_{\gamma_{2}}+\varepsilon_{C A}+2 k_{\mathrm{B}} T\left[\gamma_{2} \ln \gamma_{2}+\left(1-\gamma_{2}\right) \ln \left(1-\gamma_{2}\right)\right] \text {. }
$$

On imposing the requirement $\partial \widetilde{F}_{\mathrm{b}} / \partial \gamma_{2}=0$ and introducing an order parameter $\psi \equiv \gamma_{2}$ one obtains the equilibrium condition

$$
\frac{E(\psi)+\psi E^{\prime}(\psi)}{k_{\mathrm{B}} T}=-\ln \left(\frac{\psi}{1-\psi}\right)
$$

which for the linear variation of $E(\psi)$ reduces to

$$
\left(\varepsilon_{0}-\lambda \psi\right) / k_{\mathrm{B}} T=-\ln [\psi /(1-\psi)],
$$

which again may be solved for $\psi=\psi\left(T ; \varepsilon_{0}, \lambda\right)$; numerical calculations are shown by curves $g$ and $h$ in Fig. 12. The break in the slope $\psi^{\prime}(T)$ is characteristic of the occurrence of second order transitions. Again, Eq. (42b) rationalizes the Strässler-Kittel relation for second order transitions. The theory of Ref. [23] shows that the form of (42) is a sufficient condition for the occurrence of a second order transition.

The very simple formalism sketched above is thus capable of rationalizing a large body of information relating to $\mathrm{Fe}_{3(1-\delta)} \mathrm{O}_{4}$ and by extension, to $\mathrm{Fe}_{3-x} \mathrm{Zn}_{x} \mathrm{O}_{4}$ and to $\mathrm{Fe}_{3-y} \mathrm{Ti}_{y} \mathrm{O}_{4}$. One can in fact test the adequacy of the approach by the following fairly stringent comparisons with experimental data. The entropy of the first order transition may be calculated by substituting for $\psi=2 \beta_{1}$ the particular values $\psi_{2}$ and $\psi_{1}$ read off from Fig. 12 just above and just below the Verwey transition discontinuity and inserting these quantities into the multiplier of $k_{\mathrm{B}} T$ of Eq. (39). One finds $\widetilde{S}_{\mathrm{b}}=-k_{\mathrm{B}}\left[\psi_{2} \ln \psi_{2}-\psi_{1} \ln \psi_{1}+\left(1-\psi_{2}\right) X \ln \left(1-\psi_{2}\right)-\right.$ $\left.\left(1-\psi_{1}\right) \ln (1-\psi)+\left(\psi_{1}-\psi_{2}\right) \ln 2\right]$. This leads to the solid curve shown in Fig. 13; 
the open circles are the experimental data obtained from the latent heat measurements. A second facet involves the determination of the Seebeck coefficient $\alpha$.

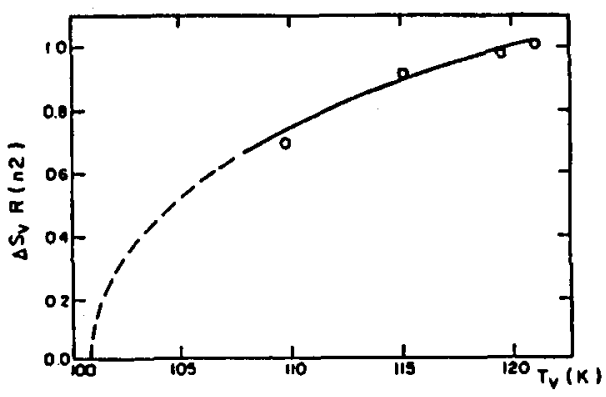

Fig. 13. Calculated variation of the entropy of transition $S_{\mathrm{v}}$ in units of $R \ln 2$ at $T=T_{\mathrm{V}}$, with $\delta$. Circles represent experimental data cited in Ref. [21].

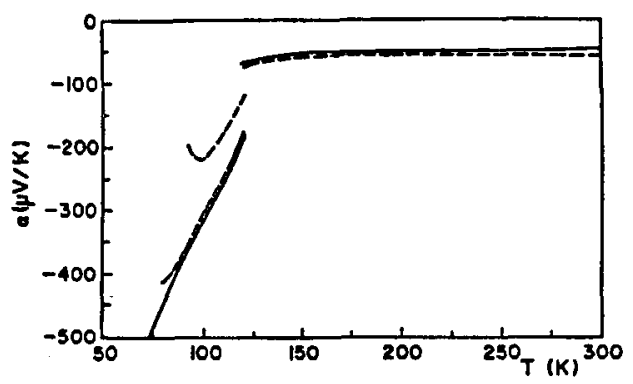

Fig. 14. Change of Seebeck coefficient with temperature for $\mathrm{Fe}_{3} \mathrm{O}_{4}, T_{V}=121.1 \mathrm{~K}$. Solid curve: theory. Dashed curves: experimental results. For high-purity material cooled in a magnetic field the agreement with theory is very good; no adjustment of parameters was required.

As is well established, as long as the kinetic contributions to $\alpha$ is small, as is the case for nearly localized carriers $\alpha=-\mu_{E} / k_{\mathrm{B}} T$, where $\mu_{E} \equiv\left(\partial \widetilde{F}_{\mathrm{b}} / \partial \gamma_{2}\right)_{T}-E$ is the chemical potential relative to the transport level at energies $E$ above the ground state $\varepsilon_{C A}$. Lack of space precludes a discussion of the requisite steps which are detailed in Ref. [24] for the determination of $\mu_{E}$ from the data; no additional parametrization is needed. Calculations lead to the variation of $\alpha$ with $T$ for $\mathrm{Fe}_{3} \mathrm{O}_{4}$ which is given by the full curve in Fig. 14, while the experimental data are shown by the dashed curve. The agreement between theory and experiment is satisfactory. Third, one may compare the resistivities. Here one needs to postulate the variation of resistivity with temperature in the limit of a small polaron model. One must fit the experimental results by introducing a third parameter that anchors the calculated resistivity to one experimental value by the procedure specified in Ref. [24]. This adjusts the magnitude of the resistivity discontinuity at $T=T_{\mathrm{V}}$. 


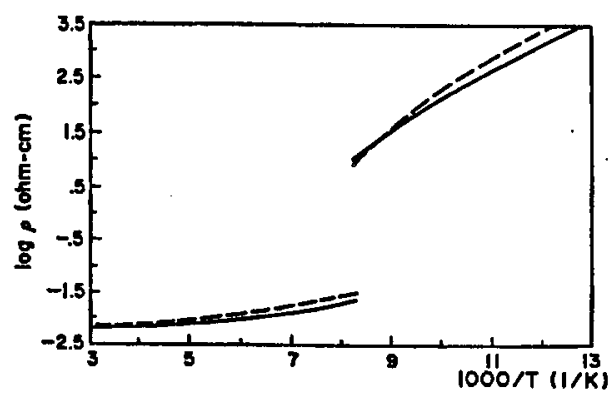

Fig. 15. Variation of $\log \rho$ vs. $1 / T$ for $\mathrm{Fe}_{3} \mathrm{O}_{4}, T_{V}=121 \mathrm{~K}$. Solid curve: theory. Dashed curve: experimental results.

The ensuing $T$ dependence is fixed by the current model. Theory and experiment are compared in Fig. 15; again, the agreement between calculated and observed resistivities is very good.

\section{Conclusion}

In summary, three rather different systems have been explored for which electron correlation phenomena in different degrees have been invoked for a rationalization of a large body of experimental data. The exposition is meant to be didactic in showing how to proceed when one-electron theories are no longer adequate to cope with experimental findings.

\section{Acknowledgments}

This research was supported on NSF grant DMR 89-21293 and on DOE grant DE-FG02-90ER 45427. The author wishes to acknowledge his deep gratitude for the many coworkers in his laboratory who undertook the painstaking research effort that led to a body of experimental data requiring interpretation. He is singularly indebted to Professor Józef Spalek who first introduced him to the theory of electron correlation phenomena and who is responsible for generating many of the requisite theories for dealing with the data presented above. The construction and application of these concepts toward a successful interpretation of the experimental work constitutes a tribute to his abilities. Extensive discussions with If. Kloor have been very helpful in identifying the use of the order disorder model in the analysis of the magnetite data.

\section{References}

[1] J.M. Honig, L.L. Van Zandt, in: Annual Review of Materials Science, Eds. R.A. Huggins, R.H. Bube, R.W. Roberts, Vol. 5, Annual Reviews, Palo Alto 1975, p. 225 and references therein to earlier reviews. 
[2] J.M. Honig, D.J. Buttrey, in: The Metallic and Nonmetallic States of Matter, Eds. P.P. Edwards, C.N.R. Rao, Taylor and Francis, London 1985, p. 261; J.M. Honig, J. Spałek, in: Advances in Solid State Chemistry, Ed. C.N.R. Rao, Indian National Science Academy, New Delhi 1986, p. 223; J.M. Honig, Proc. Indian Acad. Sci. Chem. Sci. 36, 391 (1986); M. Yethiraj, J. Solid State Chem. 80, 53 (1990); N.F. Mott, Festkörperprobleme 19, 331 (1979); N.F. Mott, Metal-Insulator Transitions, 2nd ed., Taylor and Francis, London 1990; J.B. Goodenough, Prog. Solid State Chem. 5, 145 (1971).

[3] H. Kuwamoto, J.M. Honig, J. Appel, Phys. Rev. B 22, 2626 (1980); S.A. Shivashankar, J.M. Honig, Phys. Rev. B 28, 5695 (1983); T.F. Rosenbaum, S.A. Carter, J. Solid State Chem. 88, 94 (1990); S.A. Carter, J. Yang, T.F. Rosenbaum, J. Spałek, J.M. Honig, Phys. Rev. B 43, 607 (1991); S.A. Carter, T.F. Rosenbaum, J.M. Honig, Phys. Rev. Lett. 67, 3440 (1991).

[4] H.R. Harrison, R. Aragón, J.E. Keem, J.M. Honig, in: Inorganic Synthesis, Ed. S.L. Holt, Vol. 22, Wiley, New York 1984, p. 43.

[5] D.B. McWhan, J.P. Remeika, Phys. Rev. B 2, 3734 (1970); D.B. McWhan, A. Menth, J.P. Remeika, W.F. Brinkman, T.M. Rice, Phys. Rev. B 7, 1920 (1973); D.B. McWhan, Phys. Rev. Lett. 22, 887 (1969); D.B. McWhan, T.M. Rice, J.P. Remeika, Phys. Rev. Lett. 23, 1384 (1969); A. Jayaraman, D.B. McWhan, J.P. Remeika, P.P. Dernier, Phys. Rev. B 2, 3751 (1970); T.M. Rice, D.B. McWhan, IBM J. Res. Dev. 14, 251 (1970); D.B. McWhan, A. Menth, J.P. Remeika, J. Phys. (Paris) 32, C1-1079 (1971).

[6] D.B. McWhan, J.P. Remeika, T.M. Rice, W.F. Brinkman, J.P. Maita, A. Menth, Phys. Rev. Lett. 27, 941 (1971); D.B. McWhan, J.P. Remeika, J.P. Maita, H. Okinaka, K. Kosuge, S. Kachi, Phys. Rev. B 7, 326 (1973).

[7] Y. Ueda, K. Kosuge, S. Kachi, T. Takada, J. Phys. (Paris) C 40, 275 (1979); Y. Ueda, K. Kosuge, S. Kachi, J. Solid State Chem. 31, 171 (1980).

[8] J. Spałek, A.M. Oleś, Physica B 86-88, 375 (1977); K.A. Chao, J. Spałek, A.M. Oleś, J. Phys. (Paris) C 20, 27 (1977); J. Spatek, A.M. Oleś, K.A. Chao, Phys. Status Solidi B 108, 329 (1981); J. Spałek, A.M. Oleś, J.M. Honig, Phys. Rev. B 28, 6802 (1983); J. Spałek, A. Datta, J.M. Honig, Phys. Rev. B 33, 4891 (1986); J. Spałek, A. Datta, J.M. Honig, Phys. Rev. Lett.59, 728 (1987); J. Spałek, M. Kokowski, J.M. Honig, Phys. Rev. B 39, 4175 (1989); J. Spałek, M. Kokowski, A. Datta, J.M. Honig, Solid State Commun. 70, 911 (1989); J. Spałek, J. Solid State Chem. 88, 70 (1990); J.M. Honig, J. Spałek, in: Solid State Physics, Eds. M.A.K.L. Dissanayake, K. Tenakone, P.A. Ileperuma, Vol. 2, Nova Sciences, Commack 1991, p. 41.

[9] W.F. Brinkman, T.M. Rice, Phys. Rev. B 2, 4302 (1971); T.M. Rice, W.F. Brinkman, in: Critical Phenomena in Alloys, Magnets, Superconductors, Eds. R.E. Mills, E. Ascher, R. Jaffee, McGraw-Hill, New York 1971, p. 593; J. Spałek, A. Datta, J.M. Honig, Phys. Rev. Lett. 59, 728 (1987); J. Spałek, M. Kokowski, J.M. Honig, Phys. Rev. B 39, 4175 (1989).

[10] A. Datta, J.M. Honig, J. Spałek, Phys. Rev. B 44, 8459 (1991).

[11] J. Spałek, A. Datta, J.M. Honig, Phys. Rev. B 33, 4891 (1986); J. Spalek, A.M. Oles', J.M. Honig, Phys. Rev. B 28, 6802 (1983).

[12] M.C. Gutzwiller, Phys. Rev. Lett. 10, 159 (1963); Phys. Rev. 137, A1726 (1965); P. Gopalan, Ph.D. Thesis, Purdue University, 1991, unpublished; J. Spalek, J. Solid State Chem. 88, 70 (1990). 
[13] J.M. Honig, J. Spałek, Proc. Indian Natl. Sci. Acad. A 52, 232 (1986); A. Datta, Ph.D. Thesis, Purdue University 1988, unpublished.

[14] J. Spalek, A. Datta, J.M. Honig, Phys. Rev. Lett. 59, 728 (1987); A. Datta, Ph.D. Thesis, Purdue University 1988, unpublished.

[15] H.V. Keer, H.L.C. Barros, B.L. Dickerson, T. Barfknecht, J.M. Honig, Mater. Res. Bull. 12, 137 (1977).

[16] R.A. Mohan Ram, L. Ganapathi, P. Ganguly, C.N.R. Rao, J. Solid State Chem. 63, 139 (1986); P. Ganguly, N.Y. Vasanthacharya, C.N.R. Rao, P.P. Edwards, J. Solid State Chem. 54, 400 (1984); K.P. Rajeev, N.Y. Vasanthacharya, A.K. Raychaudhuri, P. Ganguly, C.N.R. Rao, Physica C 153-155, 1331 (1988); J.G. Bednorz, K.A. Müller, Rev. Mod. Phys. 60, 585 (1988); K.P. Rajeev, G.V. Shivashankar, A.K. Raychaudhuri, Solid State Commun. 79, 591 (1991); J. B. Torrance, P. Lacorre, A.I. Nazzal, E.J. Ansaldo, Ch. Niedermayer, Phys. Rev. B 45, 8209 (1992); P. Lacorre, J.B. Torrance, J. Pannetier, A.I. Nazzal, P.W. Wang, T.C. Huang, J. Solid State Chem. 91, 225 (1991); K. Sreedhar, M. McElfresh, D. Perry, D. Kim, P. Metcalf, J.M. Honig, submitted for publication; K.S. Sreedhar, J.M. Honig, M. Darwin, M. McElfresh, P.M. Shand, J. Xu, B.C. Crooker, J. Spałek, Phys. Rev. B 46, 6382 (1992).

[17] C.J. Pethik, G.M. Carneiro, Phys. Rev. A 7, 304 (1973); J.W. Rasul, T. Li, J. Phys. C 21, 5119 (1988); T. Li, P. Wölfle, Z. Phys. 78, 45 (1990).

[18] M.C. Gutzwiller, Phys. Rev. 134, A923 (1969); Phys. Rev. 137, A1726 (1965); W.F. Brinkman, T.M. Rice, Phys. Rev. B 2, 4302 (1970); K. Seiler, C. Gros, T.M. Rice, K. Ueda, D. Vollhardt, J. Low Temp. Phys. 64, 195 (1986).

[19] J. Spałek, M. Kokowski, J.M. Honig, Phys. Rev. B 39, 4175 (1989); J. Spałek, M. Kokowski, A. Datta, J.M. Honig, Solid State Commun. 70, 911 (1989).

[20] W. Yourgrau, A. van der Merwe, G. Raw, Treatise on Irreversible and Statistical Thermophysics, McMillan, New York 1966, p. 131.

[21] J.P. Shepherd, J.W. Koenitzer, R. Aragón, C.J. Sandberg, J.M. Honig, Phys. Rev. $B$ 31, 1107 (1985); J.P. Shepherd, R. Aragón, J.W. Koenitzer, J.M. Honig, Phys. Rev. B 32, 1818 (1985); R. Aragón, R.J. Rasmussen, J.P. Shepherd, J.W. Koenitzer, J.M. Honig, J. Magn. Magn. Mater. 54-57, 1335 (1986); J.W. Koenitzer, P.H. Keesom, J.M. Honig, Phys. Rev. B 39, 6231 (1989); Z. Kąkol, J.M. Honig, Solid State Commun. 70, 967 (1989); P. Wang, Z. Kakol, M. Wittenauer, J.M. Honig, Phys. Rev. $B$ 42, 4553 (1990); J.P. Shepherd, J.W. Koenitzer, R. Aragón, J. Spałek, J.M. Honig, Phys. Rev. B 43, 8461 (1991); Z. Kąkol, J. Sabol, J. Stickler, J.M. Honig, Phys. Rev. B 46, 1975 (1992).

[22] J.M. Honig, J. Spałek, J. Less-Common Met. 156, 423 (1989); J.M. Honig, J. Spałek, P. Gopalan, J. Am. Ceram. Soc. 73, 3225 (1990); J.M. Honig, J. Spałek, J. Solid State Chem. 96, 115 (1992).

[23] S. Strässler, C. Kittel, Phys. Rev. 139, A758 (1965).

[24] R. Aragón, J.M. Honig, Phys. Rev. B 37, 209 (1988)); J.M. Honig, Phys. Chem. Miner. 15, 476 (1988). 